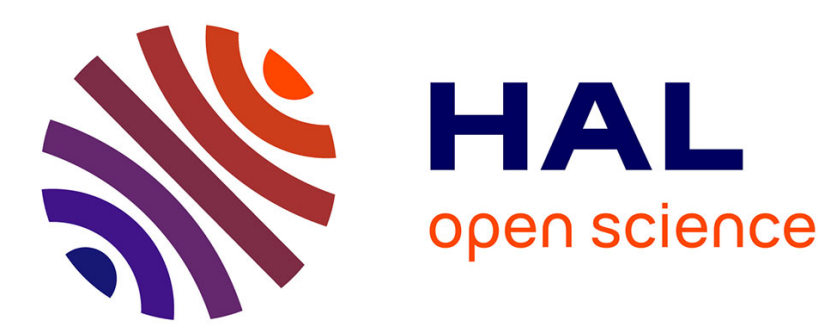

\title{
Effect of Film Cooling on Entropy Noise Generation in a Stator Blade Row
}

\author{
Thomas Bach, Maxime Huet, Robert Watson, Stephen Spence, Yasser
}

Mahmoudi

\section{- To cite this version:}

Thomas Bach, Maxime Huet, Robert Watson, Stephen Spence, Yasser Mahmoudi. Effect of Film Cooling on Entropy Noise Generation in a Stator Blade Row. AIAA Journal, 2021, 59 (8), pp.30713086. 10.2514/1.J060077 . hal-03368443

\section{HAL Id: hal-03368443 https://hal.science/hal-03368443}

Submitted on 6 Oct 2021

HAL is a multi-disciplinary open access archive for the deposit and dissemination of scientific research documents, whether they are published or not. The documents may come from teaching and research institutions in France or abroad, or from public or private research centers.
L'archive ouverte pluridisciplinaire HAL, est destinée au dépôt et à la diffusion de documents scientifiques de niveau recherche, publiés ou non, émanant des établissements d'enseignement et de recherche français ou étrangers, des laboratoires publics ou privés. 


\section{递/ QUEEN'S \\ O. UNIVERSITY BELFAST

\section{Effect of Film Cooling on Entropy Noise Generation in a Stator Blade Row}

Bach, T., Huet, M., Watson, R., Spence, S., \& Mahmoudi, Y. (2021). Effect of Film Cooling on Entropy Noise Generation in a Stator Blade Row. AIAA Journal. https://doi.org/10.2514/1.J060077

\section{Published in:}

AIAA Journal

\section{Document Version:}

Peer reviewed version

Queen's University Belfast - Research Portal:

Link to publication record in Queen's University Belfast Research Portal

\section{Publisher rights}

Copyright 2021 American Institute of Aeronautics and Astronautics

This work is made available online in accordance with the publisher's policies. Please refer to any applicable terms of use of the publisher.

\section{General rights}

Copyright for the publications made accessible via the Queen's University Belfast Research Portal is retained by the author(s) and / or other copyright owners and it is a condition of accessing these publications that users recognise and abide by the legal requirements associated with these rights.

Take down policy

The Research Portal is Queen's institutional repository that provides access to Queen's research output. Every effort has been made to ensure that content in the Research Portal does not infringe any person's rights, or applicable UK laws. If you discover content in the Research Portal that you believe breaches copyright or violates any law, please contact openaccess@qub.ac.uk. 


\title{
Effect of Film Cooling on Entropy Noise Generation in a Stator Blade Row
}

\author{
Thomas Bach * \\ Queen's University Belfast, School of Mechanical and Aerospace Engineering, Belfast, BT9 5AH, UK \\ Maxime Huet ${ }^{\dagger}$ \\ DAAA, ONERA, Université Paris Saclay, F-92322 Châtillon, France \\ Rob Watson ${ }^{\ddagger}$ and Stephen Spence ${ }^{\S}$ and Yasser Mahmoudi ${ }^{\text {Tl }}$ \\ Queen's University Belfast, School of Mechanical and Aerospace Engineering, Belfast, BT9 5AH, UK
}

In this study the effect of film cooling on entropy wave attenuation and indirect entropy noise generation was investigated in a subsonic stator blade row with a leading edge, pressure side, and suction side cooling geometry. The investigation was conducted on a small blade midspan section (in a linear cascade arrangement), using a three dimensional unsteady Reynoldsaveraged Navier-Stokes simulation approach. A planar entropy wave was injected at the inlet for a range of frequencies from $200 \mathrm{~Hz}$ to $1000 \mathrm{~Hz}$. Additionally, the compact model by Cumpsty and Marble was extended to account for a cooling flow. Results show that for an increase in coolant mass flow rate of $3.4 \%$, the entropy noise increases by about $12 \%$ for the transmitted pressure wave, while the reflected pressure wave increases by about $9 \%$. This is attributed to the change in convective acceleration in the blade passage. The film cooling was found to have a minor effect in promoting the entropy wave attenuation. The extended compact model was capable of capturing both the entropy wave attenuation and increase in the acoustic transfer function due to film cooling.

\section{Nomenclature}

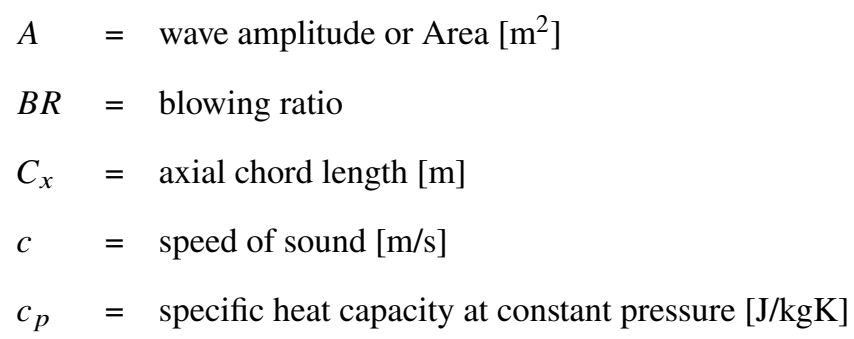

\footnotetext{
*PhD Student, School of Mechanical and Aerospace Engineering, tbach01@qub.ac.uk

${ }^{\dagger}$ Research Scientist, Department of Aerodynamics, Aeroelasticity and Acoustics, ONERA - the French Aerospace Lab, maxime.huet@ onera.fr

†Lecturer, School of Mechanical and Aerospace Engineering, r.watson@qub.ac.uk

§Professor, School of Mechanical and Aerospace Engineering, s.w.spence@qub.ac.uk

"Lecturer, School of Mechanical and Aerospace Engineering, s.mahmoudilarimi@qub.ac.uk
} 


$$
\begin{aligned}
& f \quad=\text { frequency }[\mathrm{Hz}] \\
& I=\text { momentum flux ratio } \\
& K=\text { dimensionless wave vector } \\
& M=\text { Mach number } \\
& \dot{m}=\text { mass flow rate }[\mathrm{kg} / \mathrm{s}] \\
& p=\text { pressure }[\mathrm{Pa}] \\
& s \quad=\text { entropy }[\mathrm{J} / \mathrm{kgK}] \\
& T=\text { temperature }[\mathrm{K}] \\
& t=\text { time }[\mathrm{s}] \\
& U=\text { velocity magnitude }[\mathrm{m} / \mathrm{s}] \\
& u=\text { axial velocity component }[\mathrm{m} / \mathrm{s}] \\
& v \quad=\text { velocity in } y \text {-direction }[\mathrm{m} / \mathrm{s}] \\
& w \quad=\quad \text { wave or velocity vector } \\
& \gamma=\text { ratio of specific heats } \\
& \eta \quad=\quad \text { adiabatic efficiency } \\
& \theta \quad=\text { flow angle } \\
& \lambda=\text { wavelength [m] } \\
& \rho \quad=\text { density }\left[\mathrm{kg} / \mathrm{m}^{3}\right]
\end{aligned}
$$




\section{Introduction}

Air travel is resilient to global crises, and, despite increasingly strict noise emission regulations, it is continuously growing at a rate of $4.3 \%$ annually - doubling every 15 years [1]. This compels researchers to develop new engine technologies to reduce engine noise. Fan and jet noise have been considerably reduced by introducing complex fan geometry, acoustic liners, and by increasing the bypass ratio of turbofan engines. Hence, combustion noise has emerged as the third dominant noise source [2]. Advances in the combustion process have led to increasingly unsteady combustion. This unsteadiness creates pressure waves — referred to as direct noise [3]. However, it also creates vorticity and entropy fluctuations, as well as fluctuations in mixture composition [4].

When these fluctuations are accelerated through the first turbine stage, additional pressure waves are generated, which are referred to as indirect noise [5]. The quantification of indirect noise is important, as it contributes significantly to the total combustion noise [2]. The contribution of noise due to the acceleration of vorticity fluctuations at low mean flow Mach number is small, approximately two orders of magnitude smaller than the direct noise [2]. The effect of the mixture composition on indirect noise directly depends on the combustion regime, operating conditions, and product-gas composition [6]. It is suggested that the impact of perturbations in the mixture composition is important for advanced low-emission combustors with supersonic flows, and can reach amplitudes comparable to the direct noise and entropy noise [7]. Without considering the contribution of noise due to the mixture composition, the entropy noise is believed to be the main mechanism of noise generation downstream of the combustion chamber [8]. It also has been shown that, without considering entropy wave attenuation in the turbine stage, engine noise is over predicted by almost two orders of magnitude [9]. Thus, understanding the fundamentals of entropy noise generation, entropy wave attenuation, and the techniques to reduce it, is of utmost importance.

Cumpsty and Marble [5] developed an analytical tool for indirect noise prediction in a compact turbine stage. The compact model uses the compact blade row characterised through the inlet and outlet Mach number along with the inlet and outlet flow angles. The response of the blade row is then evaluated for an incoming entropy and vorticity wave as well as pressure waves. The compact model assumes conservation of the entropy perturbation [5]. For planar entropy waves, the difference of the compact model to a two-dimensional Euler simulation was between $12-38 \%$, whilst for localized entropy disturbances the errors were much larger [10]. The accuracy of the indirect noise prediction using the compact model strongly depends on the ratio between the wavelength $(\lambda)$ and the axial chord length $\left(C_{x}\right)$, and is reasonably well predicted at $\lambda / C_{x}>10$ according to Leyko et al. [11] and Bauerheim et al. [12]. Studying the entropy transport in a duct [13], showed that shear dispersion due to the velocity profile is the main reason for entropy attenuation. The entropy wave amplitude decays as a function of residence time and frequency [14]. High frequencies are more strongly damped, whilst low frequencies depend strongly on the turbulence intensity and thermal boundary [15]. To account for the entropy attenuation in a stator [11] and a full turbine stage [12], the time-delay of the entropy wave arriving at the blade outlet was considered by extracting the time-delay from a Reynolds-averaged Navier-Stokes 
(RANS) simulation. This time-delay / shear dispersion causes the entropy wave to be non-planar when it exits the blade row.

In a comparison between a 2D Large Eddy Simulation [12] (LES) and a 3D LES [16] of a full turbine stage, a significant reduction in entropy transfer function is observed for the 3D case. The transmitted acoustic wave at the forcing frequency stays in reasonable agreement between the two simulations [16]. The reflected acoustic wave shows a slight reduction, which it is suggested is due to the choked operating condition [16]. This is in agreement with investigations of entropy noise in turbine blades with a shock, showing that pressure waves created downstream of the sonic line cannot travel upstream [17]. While previous studies have only considered planar entropy waves, Becerril et al. [18] investigated localized entropy wave streaks. These localized waves are less sensitive to shear dispersion and act like a passive scalar advected along the streamlines [18].

Previous research of entropy noise in turbine blades [11, 12, 16, 17, 19] has not yet considered the effect film cooling on entropy wave convection. Typically, turbine entry temperatures are very high (exceeding $1850 \mathrm{~K}$ for take-off conditions), requiring sophisticated cooling designs [20]. External cooling is used to create a protective layer of cool air around the turbine blade by ejecting bleed air through small holes at the leading edge, suction side, and pressure side of the blade. The effect of such a cooling flow was investigated experimentally in a nozzle flow with a perforated liner upstream of the nozzle and downstream of the entropy injection point [21]. This showed that the entropy noise is reduced by up to $11 \%$ along with up to $6 \%$ reduction in the induced temperature fluctuation [21]. However, a better understanding of the underlying mechanism is required to understand the effect of such cooling flows on entropy noise in turbine blades.

Therefore, the present study examines, for the first time, the fundamentals of entropy wave convection through a stator blade, investigating the effect of film cooling on the attenuation mechanism of entropy waves and the associated noise generation. This is investigated in a small blade mid-span section, using a three dimensional unsteady Reynolds-averaged Navier-Stokes (URANS) simulation approach. Additionally, the compact model of Cumpsty and Marble is extended to account for the effect of film cooling. For this purpose the stator blade of the axial high-pressure turbine stage (stator / rotor) at the Politecnico di Milano (PoliMi) [22] is analysed. Thereafter, a leading edge, suction side, and pressure side film cooling geometry based on the C3X test vane [23] was added to the PoliMi model. The simulations are performed for a frequency range of $200 \mathrm{~Hz}$ to $1000 \mathrm{~Hz}$, which are relevant for combustion noise [2]. The contents of this paper is as follows: Section $\Pi$ will cover the stator geometry and operating conditions along with the designed film cooling geometry. The numerical method will be described in Section III] Section IV describes the post-processing methods for the analysis of the flow field, and Section $\mathrm{V}$ covers the model validation. The impact of film cooling on the entropy wave attenuation and noise generation in a stator blade mid-span section is discussed in Section VI. Finally, in Section VII] an extension of the original compact model of Cumpsty and Marble [5] is presented. 


\section{Stator geometry}

The turbine geometry used for the analysis in this paper was an axial high-pressure turbine stage of the Politecnico di Milano (PoliMi), running at subsonic operating conditions with the rotor spinning at $7000 \mathrm{rpm}$, a static inlet pressure of $138 \mathrm{kPa}$, and a total inlet temperature of $323 \mathrm{~K}$ [22, 24, 25]. However, in this study only the stator, with an axial chord length of $C_{x}=30.6 \mathrm{~mm}$, was considered. The blade cross-section was extracted at mid-span and extruded by $3.75 \mathrm{~mm}$ (i.e. $7.5 \%$ of the blade span), and analysed in a three dimensional linear cascade arrangement. The mid-span linear cascade arrangement may impact the entropy wave attenuation when compared to an annular blade row, but the focus of the present work is on the effect of film cooling on entropy noise and not on the effect of the secondary flows such as the hub passage vortex, tip passage vortex, hub-shed vortex, and tip-shed vortex, which are all found in the PoliMi turbine [26]. Such an approach of analysing a blade in a cascade arrangement was used in previous works on film cooling [27, 28]. This assumption allows the attenuation effects due to film cooling to be distinguished, as other attenuation effects due to secondary flows are minimised. Pertinently, it also significantly reduces the mesh size, facilitating a parametric study. Taken together, the composite geometry forms an engine representative and computationally efficient model of a film cooled turbine blade.

\section{A. Film cooling geometry}

To investigate the impact of film cooling on the entropy noise, a cooling geometry was added to the turbine blade, as shown in Fig. 19. This cooling geometry is based on the C3X test vane shown in Fig. 11 p. The C3X blade has five cooling rows on the leading edge, which are referred to as showerhead (SH), two rows on the suction side (SS), as well as two rows on the pressure side (PS) of the blade [23]. As the PoliMi stator blade is much smaller when compared to the C3X test vane, the film cooling hole diameter (D) is adjusted down to $0.5 \mathrm{~mm}$, which is in agreement with blades of similar size [27]. The centre row of the SH is located on the stagnation line with the adjacent rows $(1,2$ and 4,5$)$ being staggered in spanwise direction, as shown in Fig. 1]. The spacing to diameter ratio of 7.5 [23] allowed the blade span to be reduced to $3.75 \mathrm{~mm}$ in the numerical model by making use of the periodicity in the spanwise direction. More details of the SH hole geometry are found in Table 1 The origin of the reference frame is at the blade leading edge. Hence, the first cooling row on the SS is placed at $x=8 \mathrm{~mm}$ and the first row on the PS is located at $x=7 \mathrm{~mm}$ downstream (see Fig. 11). The respective hole geometry details are identical, and can be found in Table 1 The spacing to diameter ratio was slightly increased relative to the $\mathrm{C} 3 \mathrm{X}$ geometry from 3.0 to 3.75 in order to allow for the same periodicity that was used for the SH. Consequently, the film cooling coverage is slightly reduced with less interaction between the different coolant jets [29]. Additionally, the injection angle on the PS is increased to $35^{\circ}$ in order to be consistent with the SS and with the previous 2D study by the authors looking at a blade with only PS and SS cooling [30]. 


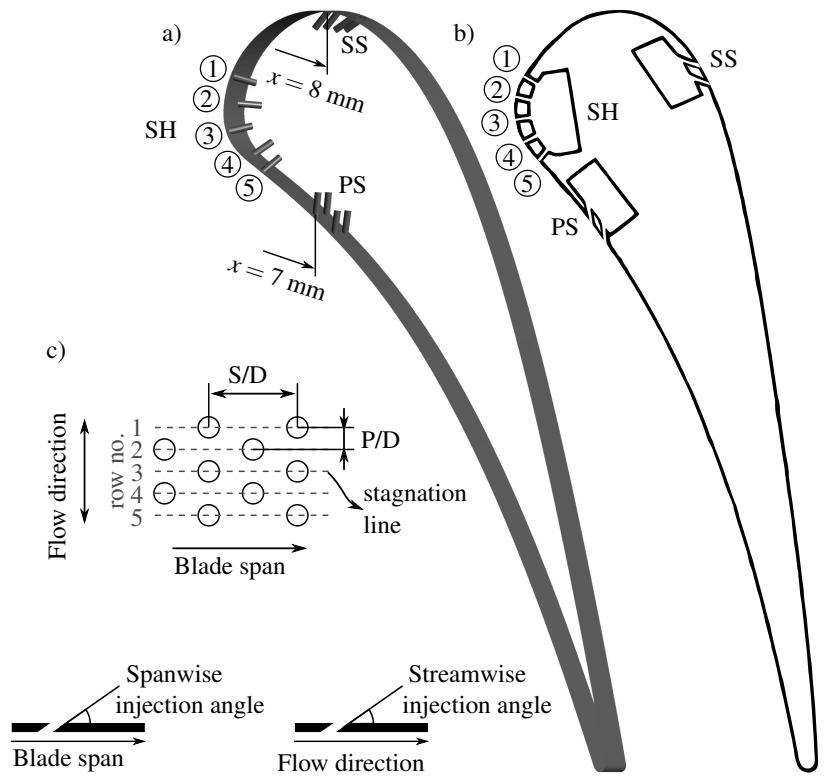

Fig. 1 a) 3D PoliMi stator blade mid-span section with film cooling, b) 2D representation of the C3X test vane [23] and c) Showerhead detail.

\section{Numerical modelling}

To analyse the effect of film cooling on the entropy wave attenuation and entropy noise generation two different computational models were developed. The first model (Case 0), has no cooling geometry and is representative of the PoliMi high-pressure stator. The second model uses the PoliMi high-pressure stator blade with a film cooling geometry similar to the C3X test vane [23]. These geometries are described in Section II.A. The sole difference between the two models is the addition of the cooling geometry and cooling inlets, whereas the domain discretization and numerical model stays unchanged. For the cooling model, the analysis was conducted at three different coolant mass flow rates. The cooling boundary conditions used in Case 2 have been chosen to match the cooling characteristics of the C3X test vane (Section III.B). For Case 1 the coolant mass flow rate was decreased by $25 \%$ with respect to Case 2 and for Case 3 the coolant mass flow rate was increased by $25 \%$ with respect to Case 2 .

\section{A. Numerical setup}

The numerical models used are based on the unsteady Reynolds-Averaged Navier-Stokes equations (URANS), which were solved in ANSYS CFX 18.2. This is a commercially available pressure-based solver using a finite volume method to discretise the Navier-Stokes equations. This approach has been used by Bake et al. [31] to investigate the Entropy Wave Generator. It considers the thermal diffusion and viscous effects which lead to entropy wave attenuation and a change in velocity gradient near the walls due to boundary layer development [30]. As turbulence closure scheme, the SST model by Menter [32] was used, as utilised in previous work investigating the PoliMi turbine [25]. The thermophysical properties of the working fluid (air) was assumed to be constant. The advective terms in the Navier-Stokes equations are 
Table 1 Film cooling hole geometry of the showerhead, and pressure and suction sides.

\begin{tabular}{|c|c|c|}
\hline $\begin{array}{c}\text { Showerhead geometric } \\
\text { parameters }\end{array}$ & $\begin{array}{l}\text { Values used in } \\
\text { this simulation }\end{array}$ & $\begin{array}{c}\text { Common } \\
\text { values [29] }\end{array}$ \\
\hline Rows of holes & 5 & \\
\hline Hole diameter, D [mm] & 0.5 & \\
\hline Pitch-to-diameter ratio (P/D) & 4.0 & $\begin{array}{l}3.0-4.0 \\
\text { up to } 7.6\end{array}$ \\
\hline Spacing-to-diameter ratio (S/D) & 7.5 & $3.0-8.0$ \\
\hline Streamwise injection angle $[\mathrm{deg}]$ & $90^{\circ}$ & \\
\hline Spanwise injection angle [deg] & $45^{\circ}$ & $20^{\circ}-45^{\circ}$ \\
\hline \multicolumn{3}{|l|}{$\begin{array}{l}\text { Downstream geometric } \\
\text { parameters (PS and SS) }\end{array}$} \\
\hline Rows of holes & 2 & \\
\hline Hole diameter, D [mm] & 0.5 & \\
\hline Pitch-to-diameter ratio (P/D) & 4.0 & \\
\hline Spacing-to-diameter ratio (S/D) & 3.75 & 3.0 up to 8.0 \\
\hline
\end{tabular}

solved using the high resolution method of ANSYS CFX. The advection scheme takes the form of:

$$
\phi_{i p}=\phi_{u p}+\beta \nabla \phi_{u p} \cdot \Delta \vec{r},
$$

where $\phi_{u p}$ is the value of the upwind node, $\vec{r}$ is the displacement vector to the integration point, and $\beta$ is a non-linear factor which takes a value $0 \leq \beta \leq 1 . \beta$ is evaluated following the Barth and Jespersen [33] limiter, which can be shown to be a Total Variation Diminishing scheme when applied to a 1D calculation [34]. The high resolution scheme computes $\beta$ to be as close as possible to 1 without introducing new extrema. If $\beta=1$ then the solution is second-order accurate. The high resolution scheme has also been used for the turbulence model equations. The time dependent term in the Navier-Stokes equations was integrated using the second-order backward Euler scheme. This is an implicit time-stepping scheme of second-order accuracy, with a fixed time step size [34].

Convergence of the inner loop has been assessed through the normalised residuals which are a measure of local imbalance of each conservative control volume equation. The convergence target level was set to $1 \times 10^{-5}$ of the root mean square of the normalised residuals. Though the fluctuations of interest are relatively small, a reduction in residual target below $1 \times 10^{-5}$ does not increase the solution accuracy. Additionally, the global imbalance across the different boundaries is monitored, allowing a maximal deviation of $1 \%$ to ensure convergence. However, the imbalance in mass, momentum, and energy are well below $1 \%$. The assessment of reaching a fully developed flow in the simulation was checked by monitoring the primitive variables such as pressure, temperature and velocity. The monitor points were located upstream and downstream of the stator blade, see location (1) and (2) in Fig. 3. The upstream pressure was last 
to reached a fully developed flow state (see Fig. 22). This state was reached once the moving average taken over $10 \mathrm{~ms}$ (i.e. the length of the FFT time period), see Fig. 2 2 , was found to change by less than $1 \%$ of the expected entropy noise amplitude over 500 time steps.
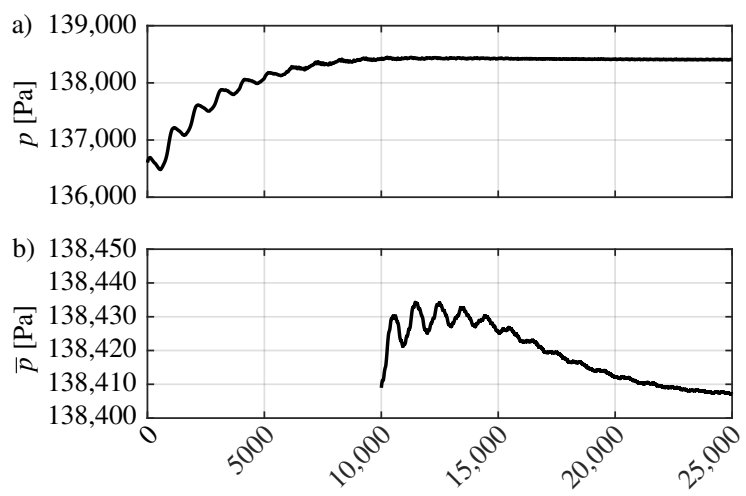

Time steps

Fig. 2 a) Upstream pressure monitor and b) moving average taken over $10 \mathrm{~ms}$ of the upstream pressure.

\section{B. Boundary conditions}

The computational domain of the stator, along with the boundary conditions (BC), are shown in Fig. 3 The stator blade row was modelled as a cascade with periodic boundary conditions in both the spanwise and pitchwise directions. The blade wall is thermally adiabatic with a no-slip condition. The assumption of an adiabatic wall is common as the thermal profiles around the blade between a conducting and adiabatic wall are nearly identical [35]. An average static pressure boundary condition was specified at the outlet to be $106.7 \mathrm{kPa}$. At the inlet a velocity of $43.45 \mathrm{~m} / \mathrm{s}$ was specified normal to the inlet plane, and a mean total temperature of $\bar{T}_{t}=323 \mathrm{~K}$ was prescribed. These $\mathrm{BC}$ correspond to the experimental setup of Knobloch et al. [24]. After a fully developed flow was reached, a planar entropy wave was imposed at the inlet. A sinusoidal temperature wave with an amplitude equal to $A=3.7 \%$ of the inlet mean total temperature $\left(\bar{T}_{t}\right)$ was forced at the inlet for the unsteady simulation:

$$
T_{t}=\bar{T}_{t}+\bar{T}_{t} A \sin (2 \pi f),
$$

where $f$ is the forcing frequency [16, 17]. When imposing a temperature fluctuation at the inlet, a pressure wave is created - often referred to as direct noise. This will interfere with the downstream generated pressure wave due to the acceleration of entropy waves. To separate the direct noise from the entropy noise, the pressure waves due to the imposed temperature fluctuations were suppressed by specifying an inlet velocity BC instead of an inlet total pressure BC. The initial turbulence was set to zero to prevent excessive entropy wave attenuation in the extended inlet duct. The extended inlet was designed to create an effective acoustic non-reflective boundary condition (NRBC), as the NRBC option in the ANSYS CFX software was found to cause the calculation to diverge at the inlet. The inlet extension is 


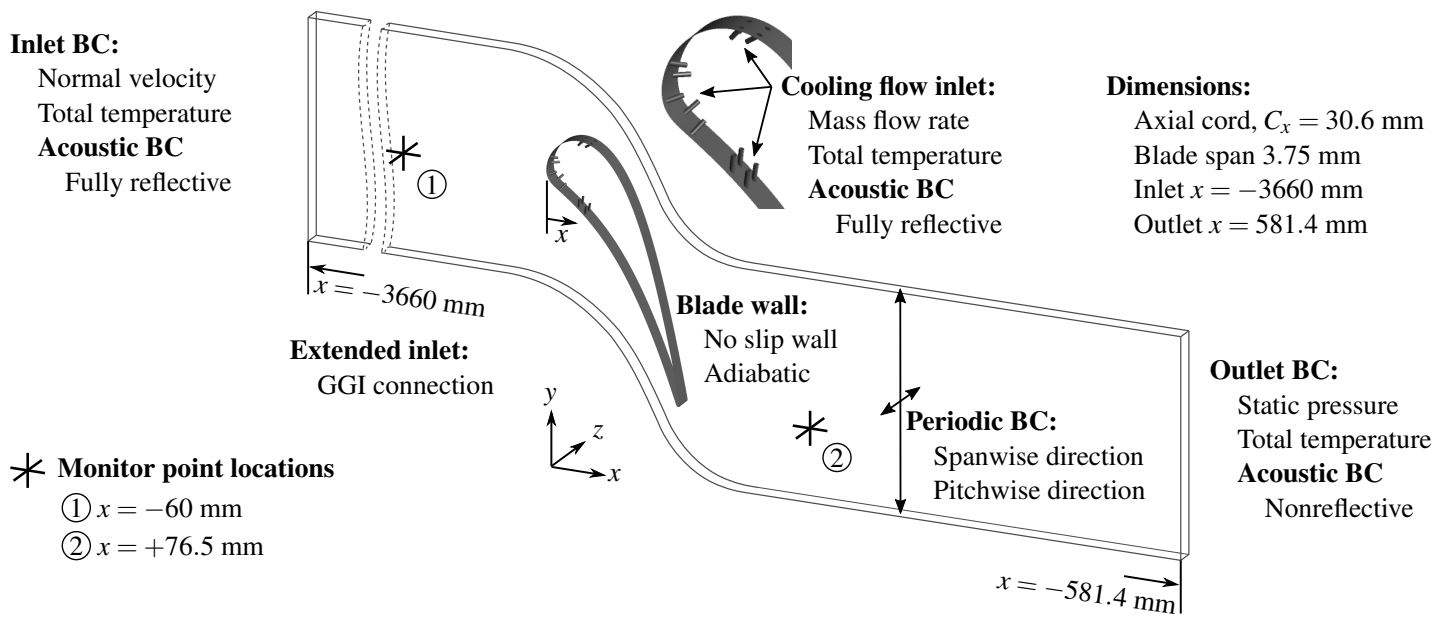

Fig. 3 Boundary conditions of stator domain.

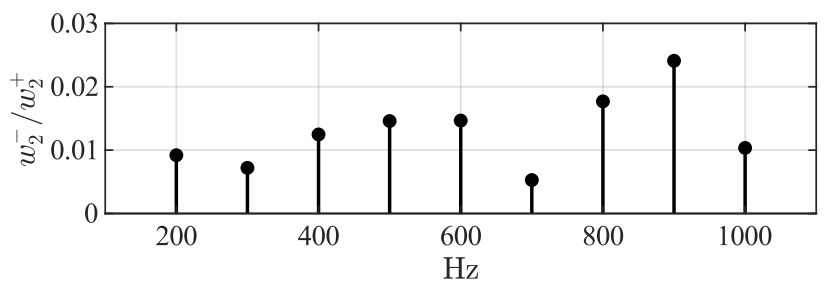

Fig. 4 Reflections of the outlet for the forcing frequencies.

quasi one-dimensional with a fine mesh resolution in the flow direction but only very few cells in the spanwise and pitchwise directions. This is sufficient, as no post-processing is undertaken in the inlet extension. The sole purpose of this duct is to mimic a NRBC and convect the planar entropy waves to the stator region. The length of the inlet duct was based on the lowest frequency of interest $(200 \mathrm{~Hz})$ and the speed of sound $(\approx 360 \mathrm{~m} / \mathrm{s})$. The acoustic wavelength of $200 \mathrm{~Hz}$ is $1.8 \mathrm{~m}$ long. Therefore, with an extended inlet length of $3.6 \mathrm{~m}$, four waves can be forced and interact with the stator blade row generating entropy noise, before the entropy noise reflections from the inlet reach the stator. Hence, any post-processing needs to be done within the $20 \mathrm{~ms}$ before the reflections reach the stator. This inlet extension was connected via a general grid interface (GGI) to the stator domain inlet. At the domain outlet the NRBC implemented available in ANSYS CFX was utilised without apparent instability. Figure 4 shows the negligible presence of spurious reflections from the outlet at the forcing frequencies with respect to the outlet pressure wave, $w_{2}^{-} / w_{2}^{+}$, for Case 0 . The definition of the pressure wave is found in Section $[\mathrm{IV}$, Equation 5 .

For the simulations with film cooling (Case 1-3) the global BC were the same as for Case 0 . The additional inlets of the cooling plenums were defined by the cooling mass flow rate and the total temperature. The experimental test case 44344 of the $\mathrm{C} 3 \mathrm{X}$ test vane has been taken as a reference. The coolant to freestream temperature ratio $\left(T R=T_{c} / T_{\infty}\right)$ of 0.86 was applied to the SH, SS, and PS [23]. $(\cdot)_{c}$ denotes the cooling flow quantities taken at the coolant plenum inlet and $(\cdot)_{\infty}$ denotes the local freestream quantity. The cooling mass flow rate has been adjusted until the blowing ratio: 
Table 2 Mass flow boundaries.

\begin{tabular}{cc}
\hline & $\dot{m}_{c}[\mathrm{~kg} / \mathrm{s}]$ \\
\hline SH 1 & $3.20 \times 10^{-5}$ \\
SH 2 & $1.70 \times 10^{-5}$ \\
SH 3 & $7.80 \times 10^{-6}$ \\
SH 4 & $1.10 \times 10^{-5}$ \\
SH 5 & $1.44 \times 10^{-5}$ \\
PS & $1.787 \times 10^{-5}$ \\
SS & $4.625 \times 10^{-5}$ \\
\hline
\end{tabular}

Table 3 Blowing ratio comparison between CFD and experiment.

\begin{tabular}{lccc}
\hline & $B R_{C F D}$ & $B R_{\text {exp }}$ & Error [\%] \\
\hline $\mathrm{SH}_{\text {ave }}$ & 1.890 & 1.959 & -3.52 \\
$\mathrm{PS}_{\text {ave }}$ & 1.617 & 1.695 & -4.58 \\
$\mathrm{SS}_{\text {ave }}$ & 0.740 & 0.777 & -4.72 \\
\hline
\end{tabular}

$$
B R=U_{c} \rho_{c} / U_{\infty} \rho_{\infty}
$$

was in agreement with the experiment. The freestream temperature was equal to the mean inlet temperature. The freestream density was evaluated at a distance of $-20 \mathrm{~mm}$ upstream of the stator leading edge. To evaluate the local freestream velocity, Bernoulli's equation was used to calculate the local freestream velocity using the upstream pressure and velocity. Though this raises concerns as the flow is compressible, this methodology was used in the experiment of Hylton et al. [23]. In the experiment [23], static pressure taps were placed on either side of the SH cooling holes in the flow direction and downstream of each cooling hole on the SS and PS. The static pressure for each hole is considered to be the average of the pressure tap on either side of the holes. For the numerical simulation, this approach was adopted to evaluate the local free stream velocity. The coolant velocity was taken as the mean coolant inlet velocity and the coolant density was extracted at the coolant inlet. The resulting mass flow rates are listed in Table 2 , where the mass flow rates for the SS and PS were the same for each individual cooling hole. These mass flow boundaries resulted in the blowing ratios recorded in Table 3 . The errors with respect to the experimental $B R$ of the C3X blade are also provided in Table 3

\section{Mesh convergence}

A hexahedral mesh was generated in ANSYS ICEM 18.2. The maximum cell size for the baseline mesh - the medium density mesh — was chosen with respect to a cut-off frequency of $1000 \mathrm{~Hz}$, based on the average axial velocity upstream of the stator [31]. A time step of $2 \times 10^{-5}$ s has been chosen, with 50 sampling points per period [31]. Additionally, a grid and time refinement study on the film cooling case (Case 2) was then conducted on a coarse, 


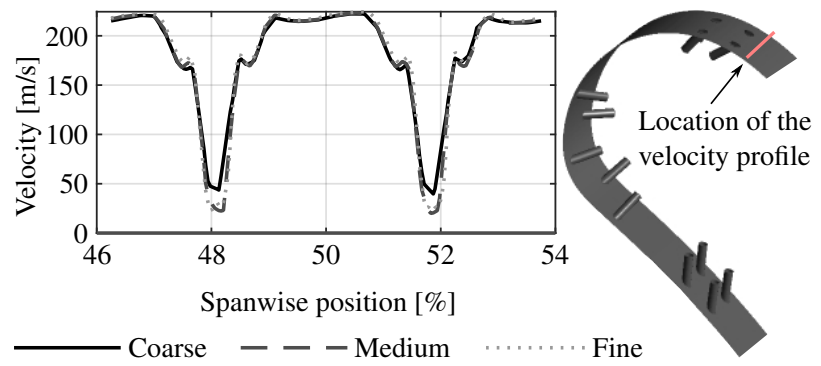

Fig. 5 Spanwise velocity distribution after the SS cooling holes for three meshes.

medium, and fine mesh at a forcing frequency of $1000 \mathrm{~Hz}$. The mesh has been doubled between the coarse to medium to fine mesh by applying the same refinement factor in all three dimensions (Table 4). The time step was decreased by a factor of 0.5 between the different meshes. The $y^{+}$value on the blade surface was approximately unity for the medium mesh, while for the coarse and fine mesh it scaled in line with the increased density. However, wall functions were applied on the inner surface of the cooling holes for computational efficiency. For the medium mesh this resulted in a $y_{\max }^{+} \approx 35$ in the cooling holes.

Table 4 Mesh characteristics for the coarse, medium, and fine meshes.

\begin{tabular}{cccc}
\hline & Elements & $\Delta t[\mathrm{~s}]$ & $y^{+}$ \\
\hline Coarse & $2 \mathrm{M}$ & $4 \times 10^{-5}$ & 1.3 \\
Medium & $4 \mathrm{M}$ & $2 \times 10^{-5}$ & 1.0 \\
Fine & $8 \mathrm{M}$ & $1 \times 10^{-5}$ & 0.8 \\
\hline
\end{tabular}

The grid study has been split into two parts. Initially, the aerodynamics were compared, and subsequently the entropy forcing parameters were checked for convergence. The acceleration is the key factor for entropy noise generation. Hence, the aerodynamic grid convergence focused on the velocity field around the stator blade. Therefore, the velocity profiles in the blade passage, in the wake (pitchwise), and near the film cooling hole outlet (spanwise) were compared. The velocity profile in the blade passage and wake were both well resolved even by the coarse mesh. Figure 5 shows the spanwise velocity profile after the SS cooling holes between the cooling flow and the blade. Very good agreement was found between the medium and fine mesh. The medium mesh was therefore used in this study in order to analyse the convection of entropy wave through the stator cascade.

With regards to entropy forcing parameters, the entropy noise transfer functions, $w_{1}^{-} / w_{1}^{s}$ and $w_{2}^{+} / w_{1}^{s}$, as well as the entropy wave transfer function, $w_{2}^{s} / w_{1}^{s}$, are considered as convergence criteria. The evaluation of these transfer functions is shown in Section IV] The respective convergence errors are listed in Table 5 The convergence error for the entropy noise transfer functions between the coarse and the fine mesh are well above 1\%, while the convergence errors between the medium and fine mesh are all within $1 \%$. Therefore the medium mesh was again chosen as the most suitable mesh for this study. 
Table 5 Convergence on transfer functions for the three meshes.

\begin{tabular}{ccc}
\hline Change in & coarse to fine & medium to fine \\
\hline$w_{1}^{-} / w_{1}^{s}$ & $-6.17 \%$ & $0.99 \%$ \\
$w_{2}^{+} / w_{1}^{s}$ & $-2.34 \%$ & $-0.49 \%$ \\
$w_{2}^{s} / w_{1}^{s}$ & $0.01 \%$ & $-0.47 \%$ \\
\hline
\end{tabular}

\section{Post-Processing methodology}

To investigate the entropy wave convection, and the resultant entropy noise transmission and reflection, data must be extracted from the simulation. To achieve this, the area averaged static pressure $(p)$, and mass flow averaged static temperature $(T)$, density $(\rho)$, and axial velocity component $(u)$ have been extracted at different $\vec{x}$ normal planes. The planes are located between $-46 \mathrm{~mm}$ (upstream) and $76 \mathrm{~mm}$ (downstream) of the stator leading edge in increments of $1 \mathrm{~mm}$. At each plane the perturbation quantities were evaluated for each time step by subtracting the mean values i.e. $p^{\prime}=p-\bar{p}$. The entropy wave $\left(w^{s}\right)$ was calculated using Equation (4) for linear perturbations, as proposed by Marble and Candel [4]. The acoustic upstream $\left(w^{-}\right)$and downstream $\left(w^{+}\right)$travelling pressure waves, based on the 1D continuity and momentum equation [4], are computed using Equation [5]:

$$
\begin{gathered}
w^{s}=\frac{s^{\prime}}{c_{p}}=\frac{p^{\prime}}{\gamma \bar{p}}-\frac{\rho^{\prime}}{\bar{\rho}}, \\
w^{ \pm}=\frac{p^{\prime \pm}}{\gamma \bar{p}}=\frac{1}{2}\left[\frac{p^{\prime}}{\gamma \bar{p}} \pm \frac{u^{\prime}}{\bar{c}}\right],
\end{gathered}
$$

where $\gamma$ is the ratio of specific heats, $\bar{c}$ is the mean speed of sound, and $s^{\prime}$ the entropy perturbation. The downstream travelling wave is denoted by $(\cdot)^{+}$and $(\cdot)^{-}$denotes the upstream travelling wave. Since plane averaged data is used instead of local values, the contribution to the entropy noise due to vortex noise becomes insignificant [2, 9]. Additionally vortex noise is likely to occur at much higher frequencies than the investigated forced frequencies i.e. $200 \mathrm{~Hz}$ to $1000 \mathrm{~Hz}$.

To analyse the entropy wave convection through the stator, the wave amplitude was evaluated by applying the Fast Fourier Transform (FFT) at each $\vec{x}$ normal plane along the axial direction to the entropy wave signal $\left(w^{s}\right)$. The FFT was taken over a time period of $0.01 \mathrm{~s}$ with a sampling frequency of $50 \mathrm{kHz}$. The upper and lower FFT limits are shown in Fig. 6. This allowed a minimum of two periods for a forced frequency of $200 \mathrm{~Hz}$ and 10 periods for a forced frequency of $1000 \mathrm{~Hz}$ to be considered. As the entropy wave convects downstream, there is a phase shift between the different planes. To ensure that the same oscillations are evaluated throughout the FFT, a phase shift of the upper and lower FFT limit is introduced. The phase shift is based on the mean axial velocity between each plane. The wave front and tail of the forced entropy wave, indicated as region (1) and (2) in Fig.6 respectively, are disregarded as they are outside the FFT limits. This is an important step in minimising errors, as the entropy wave front is slightly stretched (location (1), Fig. 6) 
Table 6 Validation of upstream and downstream mean flow properties with experimental data.

\begin{tabular}{cccc}
\hline Upstream & Experiment [24] & CFD (Case 0) & Error \\
\hline$p$ & $138 \mathrm{kPa}$ & $138.4 \mathrm{kPa}$ & $0.29 \%$ \\
$T$ & $322 \mathrm{~K}$ & $322.06 \mathrm{~K}$ & $0.02 \%$ \\
\hline Downstream & & & \\
\hline$p / p_{t, \text { ref }}$ & 0.78 & 0.76 & $-2.7 \%$ \\
Flow angle & $73.9^{\circ}$ & $75.9^{\circ}$ & $2.8 \%$ \\
\hline
\end{tabular}

and attenuated as it is convected through the extended duct. At the wave tail the entropy wave is not continuous any more (location (2), Fig. 6). The last wave amplitude overshoots and additional numerical oscillations appear. These oscillations need to be excluded from the FFT to minimize errors.

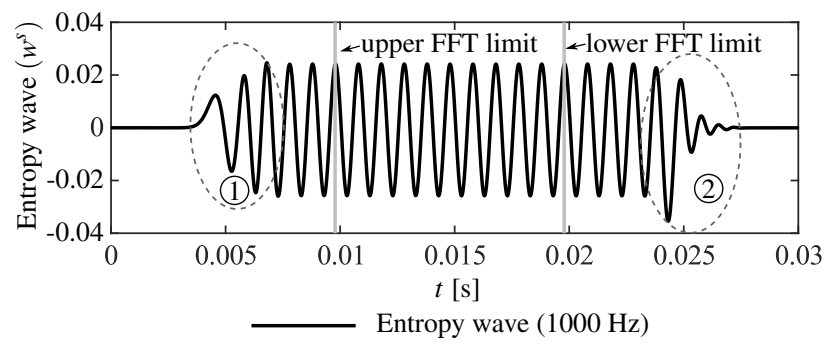

Fig. 6 Entropy wave in the time domain at $x=-46 \mathrm{~mm}$ with the FFT limiters.

Additionally, the entropy transfer functions $\left(w_{2}^{s} / w_{1}^{s}\right)$ and entropy noise transfer functions $\left(w_{2}^{+} / w_{1}^{s}\right),\left(w_{1}^{-} / w_{1}^{s}\right)$, are evaluated between a plane located at $x=-20 \mathrm{~mm}$ upstream of the vane leading edge, and $x=73.5 \mathrm{~mm}$ downstream of the stator leading edge. This will indicate to what extent the entropy waves are attenuated when travelling through the stator, and how much entropy noise is generated due to a planar entropy wave.

\section{Validation}

The aerodynamic CFD results for Case 0 , the stator blade without cooling, were validated against the experimental data from PoliMi high-pressure turbine presented by Knobloch et al. [24]. Steady mean flow data was compared at $x=-46 \mathrm{~mm}$ upstream of the leading edge, and $x=40 \mathrm{~mm}$ downstream of the leading edge. Table 6 displays the comparison of the upstream static pressure and static temperature, as well as the downstream flow angle and pressure ratio $\left(p / p_{t, r e f}\right)$, where $p_{t, r e f}$ is the total inlet pressure. The origin of the error in pressure ratio is due to an overestimation of the pressure drop across the stator blade. Since the acceleration field is key for entropy noise generation, the errors in the inlet and outlet Mach numbers have been minimised between the experiment and the CFD simulation by a slight adjustment of the inlet velocity and outlet pressure. This minimisation resulted in a $2.7 \%$ error in the pressure ratio.

Though the aerodynamics of Case 0 show good agreement with the experimental data, is is not possible to directly 
validate against the experimental acoustic data, as the stator was isolated from the turbine stage. Therefore, the acoustic transfer functions of Case 0 are compared in Fig. 77 to the analytical solution of the Cumpsty and Marble [5] model as well as the Computational Aeroacoustic (CAA) results by Emmanuelli et al. [36]. This shows that, at low frequencies, the Cumpsty and Marble [5] model results are approached, with a difference of about 6\% for the reflected and transmitted acoustic transfer functions. These differences are expected, as the criterion for the compactness of the blade stated by Leyko et al. [11] $\left(\lambda / C_{x}>10\right)$ is not met. The ratio between the entropy wavelength and the axial chord length for a frequency of $200 \mathrm{~Hz}$ is $\lambda / C_{x}=7.1$. Nevertheless, the acoustic transfer functions are in good agreement with the Cumpsty and Marble [5] model. The comparison of the present URANS simulation with the CAA model (based on an Euler simulation) by Emmanuelli et al. [36] shows a good agreement in trends of the transfer functions with respect to the forcing frequency. The over prediction has two origins. Firstly, a slightly lower inlet velocity was used by Emmanuelli et al. [36], resulting in a reduction of approximately $8 \%$ in acoustic TF according to the Cumpsty and Marble [5] model. Secondly, the boundary layer development and diffusion are disregarded in the Euler simulation by Emmanuelli et al. [36] Additionally, the present URANS simulations performed in 2D are compared with 2D LES results by Leyko et al. [11] for at similar operating conditions but with a slightly different blade geometry. Papadogiannis et al. [16] compared results of a 3D LES and a 2D LES [12] for an entropy wavelength equal to 0.4 times the stator chord length. They found that the acoustic transfer functions are very well predicted by 2D simulations and stay very similar to the 3D LES simulation. As Fig. 7p shows there is a good agreement between the acoustic URANS transfer functions from present work and the LES transfer functions by Leyko et al. [11]
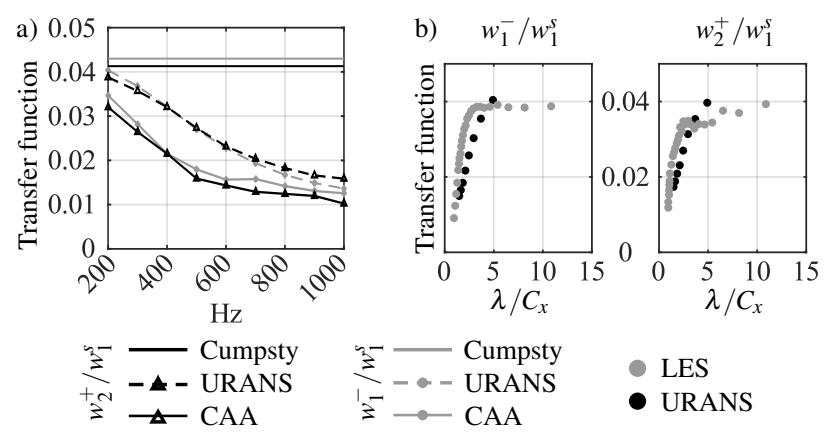

Fig. 7 a) Acoustic transfer function comparison between the Cumpsty and Marble model [5], a CAA [36] approach and the URANS results of Case 0, b) comparison between 2D URANS model with 2D LES model by Leyko et al. [11]

\section{Results}

The result section covers the analysis of the forced simulations with a forcing frequency of $200 \mathrm{~Hz}$ to $1000 \mathrm{~Hz}$. First the entropy wave convection and the main attenuations mechanisms are discussed. Thereafter, the impact of film cooling on the entropy wave attenuation for different cooling mass flow rates (Case 1-3) are discussed with respect to Case 0 (no 
cooling flow). Finally, the impact of film cooling on the entropy noise generation is discussed. Since this turbine blade originally does not contain any cooling geometry, the characteristics of the chosen cooling mass flow rates (Case 1-3) are described in the Appendix.

When entropy waves travel through a stator blade, they undergo attenuation effects and are accelerated [11]. This leads to the generation of the upstream and downstream travelling pressure waves that are referred to as entropy noise [2]. The generation of the entropy noise is visualised in Fig. 8 for Case 2 at a forcing frequency of $1000 \mathrm{~Hz}$. At $t \approx 0.08 \mathrm{~s}$ the entropy wave has not yet been accelerated in Fig. 89. Evidently, this means that there is no corresponding pressure response in Fig. $8 \mathrm{~b}$. However, when the entropy wave passes over the blade at $t \approx 0.1 \mathrm{~s}$ (see Fig. $8 \mathrm{p}$ ), the entropy wave attenuation due to shear dispersion becomes clearly visible. Additionally, the generation of pressure waves due to the entropy wave acceleration within the stator passage can clearly be seen in Fig. $8 \mathrm{~d}$.

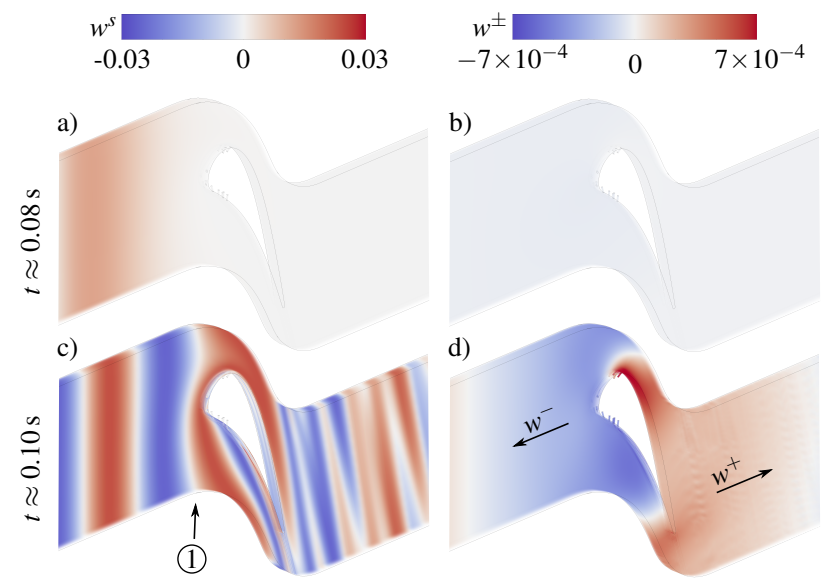

Fig. 8 Entropy wave interaction with stator blade at $t \approx 0.08 \mathrm{~s}$ and $t \approx 0.10 \mathrm{~s}$ with the corresponding pressure response.

The following section looks at the different Cases in which a planar entropy wave is forced in a frequency range from $200 \mathrm{~Hz}$ to $1000 \mathrm{~Hz}$. Firstly, the entropy wave attenuation through a stator blade is discussed (Case 0). Thereafter, the effect of film cooling on the entropy wave attenuation is shown (Case 1-3).

\section{A. Entropy wave convection}

The entropy wave convection through a stator blade has been evaluated from $x=-46 \mathrm{~mm}$ upstream to $x=76 \mathrm{~mm}$ downstream of the stator blade at an interval of $1 \mathrm{~mm}$ using the procedure described in Section IV] Figure 9 shows the entropy wave amplitude along the axial direction for three different frequencies, of $200 \mathrm{~Hz}, 600 \mathrm{~Hz}$, and $1000 \mathrm{~Hz}$. The solid line refers to the uncooled Case 0 and dotted line refers to the cooled Case 2. At location (1) in Fig. 9, the entropy wave amplitude is different for the frequencies displayed. This is due to the attenuation of the entropy wave when convecting through the long inlet duct, where high frequencies are more affected [13, 14].

As the entropy wave approaches the leading edge of the stator blade (location (2), Fig. 9), it begins to attenuate. This 
attenuation is due to shear dispersion, as the entropy wave front is subject to a varying velocity across the blade passage due to the presence of the blade leading edge. The distortion of the wave front is seen in Fig. 8 at location (1).

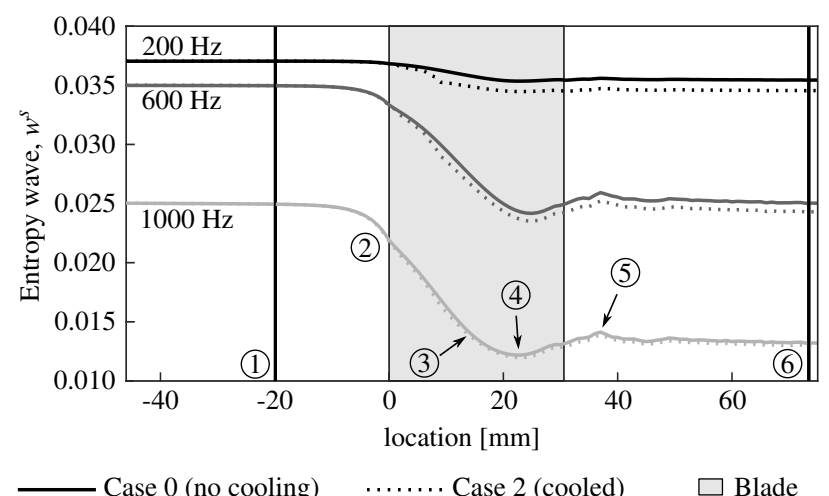

Fig. 9 Entropy wave convection in axial direction with upper (1) and lower (6) plane for the transfer function evaluation.

Within the blade passage, the attenuation rate rises for increasing frequencies (see Fig. 9). From the leading edge up to $x \approx 17 \mathrm{~mm}$ (location (3), Fig. 99 the entropy wave amplitude is linearly attenuated. Thereafter, the rate of attenuation slows, reaching a maximum attenuation at $x \approx 24 \mathrm{~mm}$ (location (4), Fig. 9). As the entropy wave convects further downstream, its amplitude again starts to increase until this ends at $x \approx 36 \mathrm{~mm}$ (location (5), Fig. 99. This increase has also been observed by Leyko et al. [11] and Becerril et al. [18].

This entropy wave attenuation profile in the blade passage can be explained with reference to the non-uniform acceleration and turning of the flow. The entropy wave undergoes a very high acceleration near the SS (location (1), Fig. 10, while on the PS the velocity change is small (location (2)). On the SS the maximum velocity is reached at $x \approx 17 \mathrm{~mm}$, indicated by the downstream dashed line (I.) in Fig. 10 The strong difference in the acceleration between the SS and PS is the cause of extensive shear dispersion, accounting for the main entropy wave attenuation. Thereafter, the entropy wave near the PS starts to accelerate significantly (location (3), Fig. 10). This slows down the rate of attenuation due to shear dispersion, and a maximum attenuation is reached at $x \approx 24 \mathrm{~mm}$, indicated by the downstream dashed line (II.) in Fig. 10

In other words, a series of particles, each associated with a sinusoidal level of entropy wave amplitude can be considered. These are initially axially co-current. But as they convect through the blade passage the particles experience a difference in acceleration, leading to a phase change between the axially distributed particles. Hence, the axial amplitude decreases. This was shown by Leyko et al. [11] who extracted a delay in arrival time of the particles at the outlet by a Lagrangian tracking of seeded particles at the inlet, to estimate the attenuation due to shear dispersion.

While the entropy wave is still strongly accelerated near the PS (location (4), Fig. 10, the velocity is constant on the SS and even slightly starts to decelerate (location (5)).

This causes a reduction of the delay time between the particles, reversing some of the phase change between the 
sinusoidal signal of each particle. Hence, leading to an increase of the axial entropy wave amplitude, previously highlighted in Fig.9 An increase in axial entropy wave amplitude in the wake has been highlighted by Beceril et al. [18]. This showed that the increase is partially due to the entropy generation of a shock wave and the wake. However, the magnitude of the generated entropy wave is of three magnitudes smaller [18] in comparison to the forced entropy wave in Fig.9. Additionally, the current simulation does not contain any shock waves as it is subsonic. Hence, the entropy generated by the shock wave and wake is be expected to be even smaller. Therefore, as any attenuation due to mixing cannot be reversed, this increase has to be due to a reverse of shear dispersion. It also becomes clear, that shear dispersion is the main attenuation mechanism within the turbine blade passage for a planar entropy wave.

At the inlet and within the blade passage, the turbulent kinetic energy is very low in comparison to the wake. Figure 9 shows that upstream of plane (1), the entropy wave amplitude is continuous and smooth. However, downstream of the trailing edge (location (5), Fig. 9), the entropy wave amplitude fluctuates and slightly decreases. This may be attributed to the high turbulence in the wake. Nevertheless, to confidently assess the effect of turbulence, a higher fidelity simulation such as LES would be required.

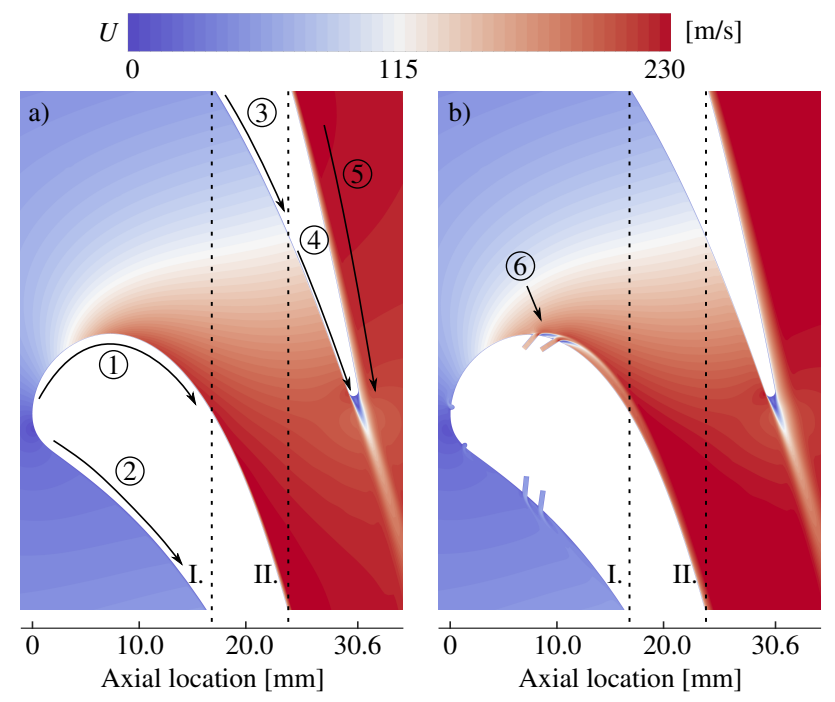

Fig. 10 Velocity field of the blade passage for a) the no cooling Case 0 and b) the film cooling Case 2 at mid-span of PS and SS cooling hole.

If a blade has a film cooling geometry, additional mass is injected into the flow near to the blade. The cooling flow injected is unperturbed, meaning it does not contain the forced entropy wave of the mainstream. The added mass is therefore available to absorb the mainstream entropy perturbations via dissipation. This can happen through both turbulent mixing with the mainstream and thermal convection. However, a long resident time is required for both attenuation mechanisms to affect the entropy wave attenuation [14]. As the cooling flow slightly displaces the mainstream, the velocity field also changes. This leads to a slight change in shear dispersion. Hence, there are multiple attenuation mechanisms responsible for the reduction in entropy wave amplitude in Fig. 9 for the cooled case (dotted 
lines) in comparison to Case 0 (solid lines).

To quantify the entropy wave attenuation due to film cooling, a comparison between the entropy wave transfer function (TF) with and without film cooling is presented. The entropy transfer function is defined as $w_{2}^{s} / w_{1}^{s}$. The location at which the entropy wave amplitude is extracted is indicated by the vertical lines (1) and (6) in Fig. 9. The change in TF is reported as a percentage change:

$$
\Delta T F=\frac{T F_{\text {film cooling }}-T F_{\text {no cooling }}}{T F_{\text {no cooling }}} \times 100
$$

where the non-film cooling case has been taken as the reference. The change in the entropy TF is evaluated for the three different cooling mass flow rates for a range of frequencies from $200 \mathrm{~Hz}$ to $1000 \mathrm{~Hz}$. In Fig. 117 the entropy wave TF for Case 0 and Case 3 are plotted, while Fig. 11p shows the percentage change due to film cooling. A reduction in entropy TF due to film cooling is evident in Fig. 11p, with a maximum attenuation at a frequency of $600 \mathrm{~Hz}$ in all three cases. Figure 11 shows that the attenuation profile of Case 0 and Case 3 are very similar. The rate of attenuation increases with increasing frequency, reaching a maximum at approximately $500 \mathrm{~Hz}$ to $600 \mathrm{~Hz}\left(\lambda / C_{x} \approx 2.9-2.3\right)$. The same finding can be found in the entropy wave transfer function data published by Leyko et al. [11]. Hence, the attenuation rate correlates with the blade size and the maximum attenuation change in Fig.11p is a result of the same mechanism. The attenuation mechanism responsible for additional attenuation due to film cooling cannot be determined with certainty, the variation in attenuation for different frequencies is likely due to shear dispersion. While the attenuation is not uniform between the frequencies, the reduction in TF scales with the global mass flow increase due to the coolant. The mass flow rate increase is $2.09 \%, 2.71 \%$ and $3.40 \%$ for Case 1 to Case 3, respectively.

This leads to the conclusion that film cooling does have the capability to attenuate the entropy wave within the stator passage. However, the attenuation due to film cooling occurs at a very small scale. A clear reduction can be seen in Fig. 11 p with very similar trends for different cases, but the percentage changes are of the same order as the numerical errors. Therefore, the global attenuation mechanisms within the blade passage clearly dominate. Nevertheless, a cooling flow has the potential to attenuate the entropy wave by approximately the same percentage as the global mass flow increases due to the coolant injection.

\section{B. Entropy noise generation}

To quantify the entropy noise generation due to an entropy wave, the acoustic transfer function (TF) are used. The acoustic transfer functions, $\left(w_{1}^{-} / w_{1}^{s}\right)$ and $\left(w_{2}^{+} / w_{1}^{s}\right)$, represent the upstream and downstream pressure wave response due to an initial entropy wave. These are plotted in Fig. $12 \mathrm{p}$ and $12 \mathrm{p}$ for Case 0 and Case 3 . The effect of film cooling on the acoustic transfer functions is shown in Figs. 12 a and $12 \mathrm{~d}$ for the reflected and the transmitted pressure wave, respectively. The comparison between the non-cooled case (Case 0) and the three different cooled cases (Case 1-3) shows a possible 

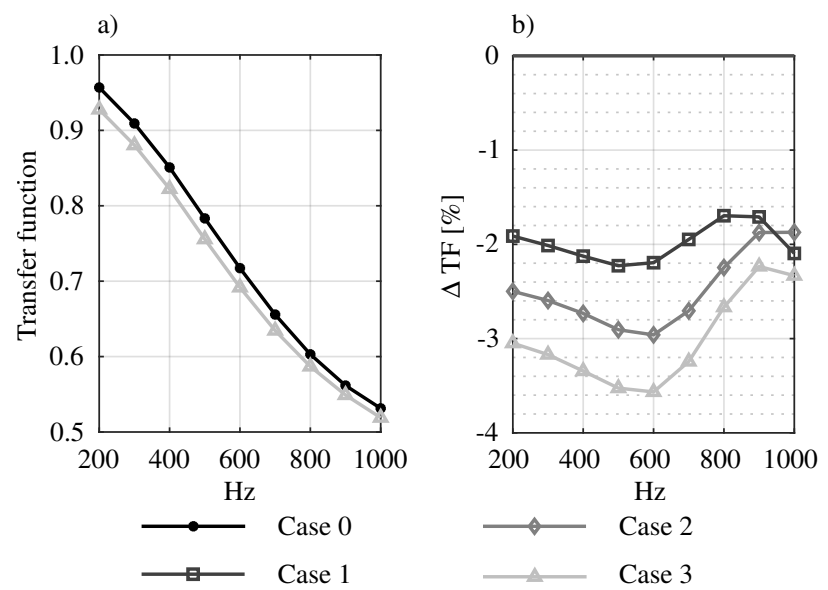

Fig. 11 a) Entropy wave transfer function for Case 0 and Case 3 and b) difference in entropy wave transfer function with respect to Case 0 for different cooling mass flow rates.

increase of over $9 \%$ for $w_{1}^{-} / w_{1}^{s}$ and $12 \%$ for $w_{2}^{+} / w_{1}^{s}$. This is a significant increase in entropy noise, considering the additional cooling mass flow for Case 3 is only 3.4\%. The maximum increase in entropy noise in Figs. 12 e and $12 \mathrm{~d}$ is found in the region between $600 \mathrm{~Hz}$ to $800 \mathrm{~Hz}$, where the entropy noise reduction rate starts to decrease in Fig. $12 \mathrm{a}$ and 12p. This closely correlates with the maximum attenuation rate of the entropy wave in Fig. 11. The peak in additional noise generation, as well as the slight shift of the maximum entropy noise with respect to the maximum entropy wave attenuation, is likely due to the geometry of the blade. Hence, it is speculated that it is due to the superposition of pressure waves generated within the stator blade i.e. a phase shift between the pressure waves generated at different axial locations. Nevertheless, film cooling increases the acoustic pressure response to an incoming entropy wave, for both the upstream and downstream travelling pressure waves.
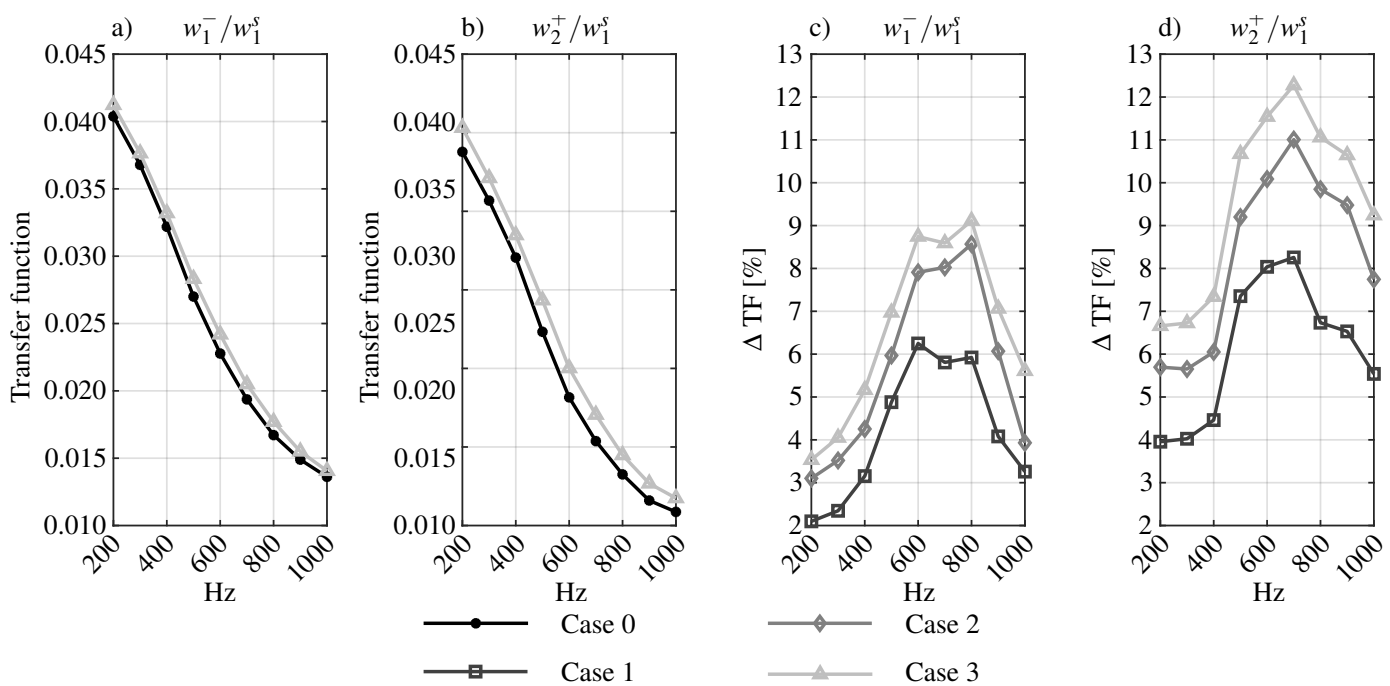

Fig. 12 a) and b) Reflected and transmitted entropy noise transfer function for Case 0 and Case 3 and c) and d) the difference in reflected and transmitted entropy noise transfer function with respect to Case 0 for different cooling mass flow rates. 
The additional noise generated can be explained by the change of the acceleration within the stator passage. This results in the generation of additional (indirect) entropy noise, as shown by Marble and Candel [4]. Figure 13a shows the difference between the no cooling case i.e. Case 0 and Case 2. The increase in velocity may not be large, but a $15 \mathrm{~m} / \mathrm{s}$ increment represents approximately $8 \%$ of the maximum velocity in the blade passage. As the coolant is injected, it stays near the blade surface. This is seen at location (1) in Fig. 13p, where the cooling flow on the SS is clearly unperturbed at the start. In the SH and PS regions of the blade, the cooling flow mixes quicker with the entropy wave. However, it still stays near the blade surface (see the Appendix for more details on the cooling flow characterisation). This essentially leads to a narrower blade passage for the entropy wave to pass through leading to a global acceleration of the bulk flow (location (2), Fig. 13 ) generating additional entropy noise. The greatest change in velocity can be observed at the coolant outlet on the SS, see location (3), Fig. 13. The adiabatic effectiveness above $70 \%$ is highlighted green in Fig. 13 . The flow within this region had only limited time to mix with the bulk flow and is therefore only slightly perturbed by the entropy wave. However, near the SS there is significant change in velocity outside this region which leads to a more localised entropy noise source [30]. On the PS and the SH cooling region the velocity change of the bulk flow is relatively small hence there are no local noise sources.
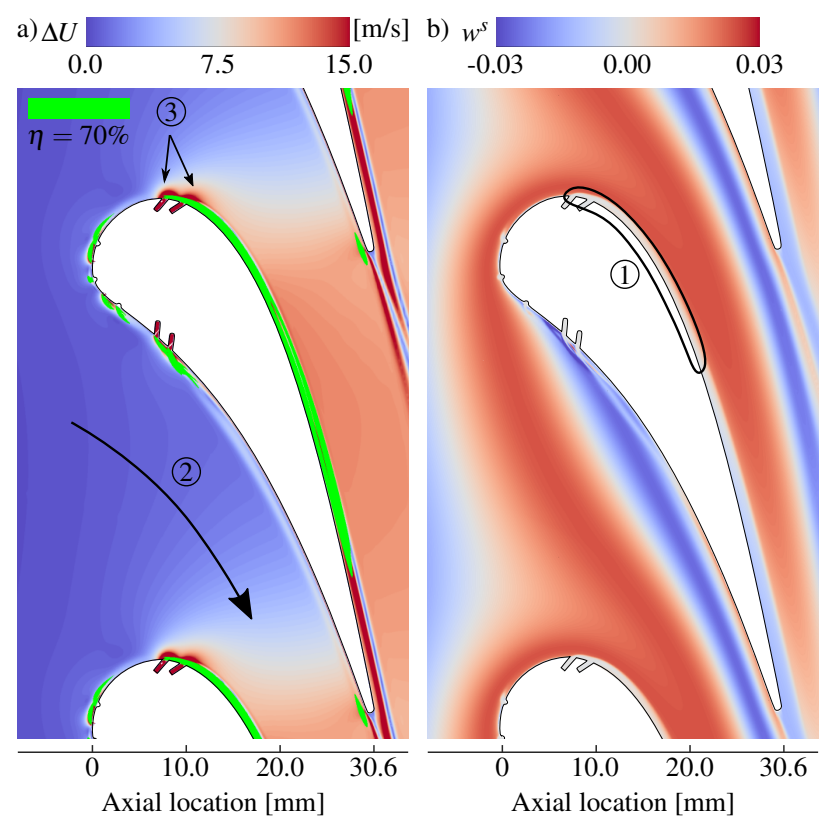

Fig. 13 a) Velocity difference between the no film cooling (Case 0) and Case 2, with indication of coolant (green) and b) the entropy wave of $1000 \mathrm{~Hz}$ for Case 2.

These findings suggest that film cooling has a minor effect on entropy wave attenuation. Instead the additional mass flow, leads to a stronger acceleration of the bulk flow and localised acceleration in the vicinity of the coolant outlet. This additional acceleration leads to an increase in entropy noise. 


\section{Compact model extension for a cooling flow}

The main application of high-fidelity numerical methods (e.g. 3D URANS) is in the research field of enhancing the understanding of fundamental physical processes. In real industrial design environments, there is an additional need for less computational-time consuming prediction tools [9, 37]. The available and widely used low order model of Cumpsty and Marble [5] accounts for the propagation of acoustic, vorticity, and entropy waves through a blade row. However, this model does not incorporate the cooling flows of the turbine blades. Modern aero engines require a cooling geometry due to the high turbine entry temperatures. The results presented in Section $\mathrm{VI}$ have shown that film cooling has significant influence on the entropy wave attenuation and entropy noise generation. Hence, there is a need for low order model of entropy noise which accounts for the effect of film cooling on the attenuation of entropy wave and the noise generation associated with it.

\section{A. Analytical developments}

Cumpsty and Marble [5] developed a model describing the propagation of entropy, vorticity, and acoustic waves through a compact blade row. The model is based on a previous model by Marble and Candel [4] for the wave propagation through a nozzle. It considers two dimensions accounting for the turning of the flow through the blade row (circumferential component) but neglecting any radial variations, which is valid for geometries with large hub-to-tip ratios. The blade spacing is assumed to be small in comparison to the axial chord length, neglecting the blade details, and only considering the inlet and outlet Mach numbers and flow angles [5] (see Fig. 14). As only the inlet and outlet velocity are of interest, the axial component, $u=w \cos \theta$, and circumferential component, $v=w \sin \theta$, can be evaluated from the velocity triangle. The velocity perturbations $u^{\prime}$ and $v^{\prime}$ can be related to $w^{\prime}$ by expressing $w$ (full velocity) as a function of $u=\bar{u}+u^{\prime}$ and $\theta=\bar{\theta}+\theta^{\prime}$, and then can be linearised. The same procedure is repeated for $v$ resulting in:

$$
\frac{u^{\prime}}{\bar{u}}=\frac{w^{\prime}}{\bar{w}}-\theta^{\prime} \tan \bar{\theta}, \quad \frac{v^{\prime}}{\bar{v}}=\frac{w^{\prime}}{\bar{w}}+\frac{\theta^{\prime}}{\tan \bar{\theta}} .
$$

The blade passage is short in comparison to the wavelength, preventing a phase shift between the upstream and downstream wave. For entropy waves to interact with the blade row, it is important that the pressure drop across the blade is large even though the perturbations are small.

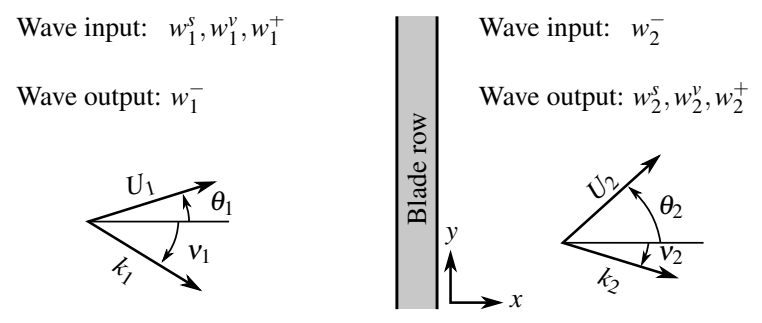

Fig. 14 Visualisation of the Compact Model with the inputs and outputs. 
The original compact model assumed (i) the conservation of entropy across the blade, indicating that the perturbations do not change the losses across the cascade, (ii) continuity of mass flow across the blade and hence no cooling flow, and (iii) conservation of stagnation enthalpy [5]. To allow for the introduction of a cooling flow, the assumptions by Cumpsty and Marble [5] are relaxed and the conservation of mass, energy (enthalpy), and entropy now read as:

$$
\begin{aligned}
\dot{m}_{1}+\dot{m}_{c} & =\dot{m}_{2}, \\
\dot{m}_{1} T_{t 1}+\dot{m}_{c} T_{t c} & =\dot{m}_{2} T_{t 2}, \\
\dot{m}_{1} s_{1}+\dot{m}_{c} s_{c} & =\dot{m}_{2} s_{2},
\end{aligned}
$$

where the subscript $(\cdot)_{1},(\cdot)_{2},(\cdot)_{c}$ and $(\cdot)_{t}$ refer to the upstream, downstream, and cooling flow, and stagnation temperature respectively. The mass flow rate, stagnation temperature, and entropy are defined as:

$$
\begin{aligned}
\dot{m} & =\rho u A \\
T_{t} & =T\left(1+\frac{\gamma-1}{2} M^{2}\right), \\
s & =c_{p} \log \frac{p^{1 / \gamma}}{\rho}
\end{aligned}
$$

respectively, where $A$ is the area normal to the $x$ direction. The fluctuation of mass flow rate and stagnation temperature have to be written as a function of the primitive variables. Therefore, the first order perturbed mass flow rate (Equation (11) ) and stagnation temperature (Equation (12)) are derived, leading to:

$$
\begin{aligned}
& \frac{\dot{m}^{\prime}}{\overline{\bar{m}}}=\frac{u^{\prime}}{\bar{u}}+\frac{\rho^{\prime}}{\bar{\rho}}, \\
& \frac{T_{t}^{\prime}}{\overline{T_{t}}}=\frac{1}{1+\frac{\gamma-1}{2} \bar{M}^{2}}\left((\gamma-1) \bar{M}^{2} \frac{w^{\prime}}{\bar{w}}+\frac{T^{\prime}}{\bar{T}}\right),
\end{aligned}
$$

with the mean flow quantities indicated by $\overline{(\cdot)}$ and the perturbations by $(\cdot)^{\prime}$. To write the perturbed mass flow rate as a function of the primitive variables, Equation (7) and Equation (13) are substituted into Equation (14). If the entropy perturbation is written in terms of temperature and pressure fluctuation, $s^{\prime} / c_{p}=T^{\prime} / \bar{T}-(\gamma-1) p^{\prime} /(\gamma \bar{p})$, the mass flow rate and stagnation temperature in terms of primitive variables become:

$$
\frac{\dot{m}^{\prime}}{\overline{\dot{m}}}=\frac{p^{\prime}}{\gamma \bar{p}}-\frac{s^{\prime}}{c_{p}}+\frac{1}{\bar{M}} \frac{w^{\prime}}{\bar{c}}-\theta^{\prime} \tan \bar{\theta},
$$




$$
\frac{T_{t}^{\prime}}{\overline{T_{t}}}=\frac{1}{1+\frac{\gamma-1}{2} \bar{M}^{2}}\left((\gamma-1) \bar{M} \frac{w^{\prime}}{\bar{c}}+\frac{s^{\prime}}{\overline{c_{p}}}+(\gamma-1) \frac{p^{\prime}}{\gamma \bar{p}}\right),
$$

respectively.

Assuming perfect mixing between the freestream and coolant, and that no fluctuations are introduced by the coolant flow (steady mass flow rate and stagnation temperature are imposed for the coolant flow), the perturbed form of Equations (8)-10] become:

$$
\begin{aligned}
& \frac{\overline{\dot{m}}_{1}}{\overline{\dot{m}}_{2}} \frac{\dot{m}_{1}^{\prime}}{\overline{\dot{m}}_{1}}=\frac{\dot{m}_{2}^{\prime}}{\overline{\dot{m}}_{2}}, \\
& \frac{\overline{\dot{m}}_{1}}{\overline{\dot{m}}_{2}} \frac{\bar{T}_{t 1}}{\bar{T}_{t 2}}\left(\frac{\dot{m}_{1}^{\prime}}{\overline{\dot{m}}_{1}}+\frac{T_{t 1}^{\prime}}{\bar{T}_{t 1}}\right)=\frac{\dot{m}_{2}^{\prime}}{\overline{\dot{m}}_{2}}+\frac{T_{t 2}^{\prime}}{\bar{T}_{t 2}}, \\
& \frac{\overline{\dot{m}}_{1}}{\overline{\dot{m}}_{2}} \frac{\bar{s}_{1}}{\bar{s}_{2}}\left(\frac{\dot{m}_{1}^{\prime}}{\overline{\dot{m}}_{1}}+\frac{c_{p}}{\bar{s}_{1}} \frac{s_{1}^{\prime}}{c_{p}}\right)=\frac{\dot{m}_{2}^{\prime}}{\overline{\dot{m}}_{2}}+\frac{c_{p}}{\bar{s}_{2}} \frac{s_{2}^{\prime}}{c_{p}} \text {. }
\end{aligned}
$$

Finally, the matrix $\left[E_{p}^{e}\right]$ relating the primitive variable perturbations at the inlet to those at the the outlet can be constructed. For this, the Kutta condition proposed by Cumpsty and Marble [5] is used:

$$
\theta_{2}^{\prime}=0
$$

or, in a more general form also proposed by Cumpsty and Marble [5]:

$$
\theta_{2}^{\prime}=\alpha \theta_{1}^{\prime}
$$

where $\alpha$ is a real constant which is to be determined experimentally. Additionally, Equations (16) and (17) are substituted into the perturbed conservation Equations (18) - 20], leading to Equation [23):

$$
\left[E_{p}^{e}\right]_{1} \cdot\left(\begin{array}{c}
s^{\prime} / c_{p} \\
w^{\prime} / \bar{c} \\
p^{\prime} / \gamma \bar{p} \\
\theta^{\prime}
\end{array}\right)_{1}=\left[E_{p}^{e}\right]_{2} \cdot\left(\begin{array}{c}
s^{\prime} / c_{p} \\
w^{\prime} / \bar{c} \\
p^{\prime} / \gamma \bar{p} \\
\theta^{\prime}
\end{array}\right)_{2}
$$

with 


$$
\begin{aligned}
& {\left[E_{p}^{e}\right]_{1}=}\left(\begin{array}{cccc}
\beta\left(c_{p} / \bar{s}_{1}-1\right) & \beta / \bar{M}_{1} & \beta & -\beta \tan \bar{\theta}_{1} \\
-\varepsilon & \varepsilon / \bar{M}_{1} & \varepsilon & -\varepsilon \tan \bar{\theta}_{1} \\
\delta\left(\mu_{1}-1\right) & \delta\left((\gamma-1) \mu_{1} \bar{M}_{1}+1 / \bar{M}_{1}\right) & \delta\left((\gamma-1) \mu_{1}+1\right) & -\delta \tan \bar{\theta}_{1} \\
0 & 0 & 0 & \alpha
\end{array}\right), \\
& {\left[E_{p}^{e}\right]_{2}=\left(\begin{array}{cccc}
c_{p} / \bar{s}_{2}-1 & 1 / \bar{M}_{2} & 1 & -\tan \bar{\theta}_{2} \\
-1 & 1 / \bar{M}_{2} & 1 & -\tan \bar{\theta}_{2} \\
\mu_{2}-1 & (\gamma-1) \mu_{2} \bar{M}_{2}+1 / \bar{M}_{2} & (\gamma-1) \mu_{2}+1 & -\tan \bar{\theta}_{2} \\
0 & 0 & 0 & 1
\end{array}\right), }
\end{aligned}
$$

and

$$
\begin{gathered}
\beta=\frac{\overline{\dot{m}}_{1} \bar{s}_{1}}{\overline{\dot{m}}_{2} \bar{s}_{2}}, \\
\delta=\frac{\overline{\dot{m}}_{1} \bar{T}_{t 1}}{\overline{\dot{m}}_{2} \bar{T}_{t 2}}, \\
\varepsilon=\frac{\overline{\dot{m}}_{1}}{\overline{\dot{m}}_{2}}, \\
\mu=1 /\left(1+(\gamma-1) \bar{M}^{2} / 2\right) .
\end{gathered}
$$

To complete the model, a few more steps are necessary, following Leyko et al. [11]. To relate the perturbations of the primitive variables to entropy $w^{s}$ and vorticity $w^{v}$ waves, as well as to the downstream $w^{+}$and upstream $w^{-}$ acoustic waves, a transformation matrix $\left[P_{w}^{p}\right]$ is required [11] :

$$
\left(\begin{array}{c}
s^{\prime} / c_{p} \\
w^{\prime} / \bar{c} \\
p^{\prime} / \gamma \bar{p} \\
\theta^{\prime}
\end{array}\right)=\left[P_{w}^{p}\right] \cdot\left(\begin{array}{c}
w^{s} \\
w^{v} \\
w^{+} \\
w^{-}
\end{array}\right),
$$

with 


$$
\left[P_{w}^{p}\right]=\left(\begin{array}{cccc}
1 & 0 & 0 & 0 \\
0 & -i \frac{\sin \left(v_{\xi}-\bar{\theta}\right)}{K_{\xi}} & \frac{K_{+} \cos \left(v_{+}-\bar{\theta}\right)}{1-\bar{M} K_{+} \cos \left(v_{+}-\bar{\theta}\right)} & \frac{K_{-} \cos \left(v_{-}-\bar{\theta}\right)}{1-\bar{M} K_{-} \cos \left(v_{-}-\bar{\theta}\right)} \\
0 & 0 & 1 & 1 \\
0 & i \frac{\cos \left(v_{\xi}-\bar{\theta}\right)}{\bar{M} K_{\xi}} & \frac{K_{+} \sin \left(v_{+}-\bar{\theta}\right)}{\bar{M}\left[1-\bar{M} K_{+} \cos \left(v_{+}-\bar{\theta}\right)\right]} & \frac{K_{-} \sin \left(v_{-}-\bar{\theta}\right)}{\bar{M}\left[1-\bar{M} K_{-} \cos \left(v_{-}-\bar{\theta}\right)\right]}
\end{array}\right) .
$$

Finally, the upstream and downstream waves can be connected through the wave vector, $\left[P_{w}^{p}\right]$ matrix and $\left[E_{p}^{e}\right]$ matrix, resulting in:

$$
\underbrace{\left[E_{p}^{e}\right]_{1} \cdot\left[P_{w}^{p}\right]_{1}}_{[B]_{1}} \cdot\left(\begin{array}{c}
w^{s} \\
w^{v} \\
w^{+} \\
w^{-}
\end{array}\right)_{1}^{[B]_{2}}=\underbrace{\left[E_{p}^{e}\right]_{2} \cdot\left[P_{w}^{p}\right]_{2}} \cdot\left(\begin{array}{c}
w^{s} \\
w^{v} \\
w^{+} \\
w^{-}
\end{array}\right)_{2} .
$$

Equation (32) is written in terms of upstream and downstream waves, hence on the left-hand side $w^{-}$is unknown whereas on the right-hand side $w^{-}$is known. Therefore if only an isolated blade row is considered, the last column of the $[B]$ matrix can be permuted from left-to-right, resulting in the matrices $[A]_{\text {in }}$ and $[A]_{\text {out }}$. Additionally, the last term of the wave vector needs to be permuted, leading to:

$$
\left(\begin{array}{c}
w_{2}^{s} \\
w_{2}^{v} \\
w_{2}^{+} \\
w_{1}^{-}
\end{array}\right)=[A]_{\text {out }}^{-1} \cdot[A]_{\text {in }}\left(\begin{array}{c}
w_{1}^{s} \\
w_{1}^{v} \\
w_{1}^{+} \\
w_{2}^{-}
\end{array}\right)
$$

In order to analyse an isolated blade row with film cooling the following input parameters are required. The blade row is defined by the inlet and outlet Mach numbers, $\bar{M}$, and flow angles, $\bar{\theta}$. To account for the coolant flow, Equation (26)-(28) need to be defined, requiring $\overline{\dot{m}}_{1}, \overline{\dot{m}}_{2}, \bar{T}_{t 1}, \bar{T}_{t 2}, \bar{s}_{1}$ and $\bar{s}_{2}$. To evaluate the downstream quantities the time-averaged form of Equations (8)-(10) should be used, as the upstream and coolant flows are uniform whereas the downstream flow is not, due to a lack of perfect mixing. Hence $\overline{\dot{m}}_{1}, \overline{\dot{m}}_{c}, \bar{T}_{t 1}, \bar{T}_{t c}, \bar{s}_{1}$ and $\bar{s}_{c}$ are extracted from a steady RANS simulation. 


\section{B. Compact model results}

The compact model extension (CM Ext.), which is capable of accounting for a cooling flow is validated against the CFD results of Case 2 and the original Cumpsty and Marble [5] model in Fig. 15] It is important to note that the CFD results at $200 \mathrm{~Hz}$ are not compact, hence a certain degree of discrepancy is expected. Nevertheless, Fig. [15] shows that as the frequency decreases, the CFD results are approaching the $0 \mathrm{~Hz}$ results of the extended compact model. This is found to be true for both the entropy wave and entropy noise TFs.

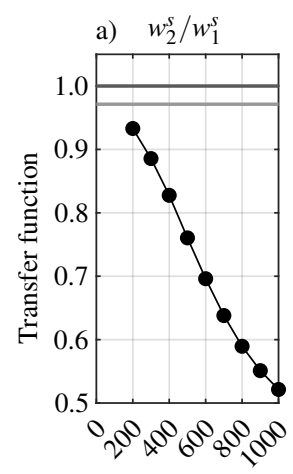

$\mathrm{Hz}$

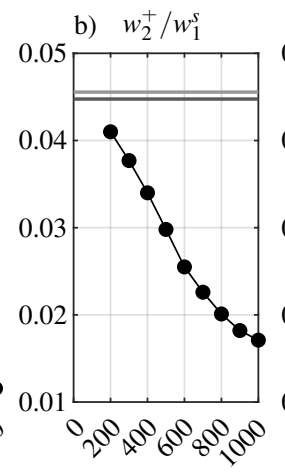

$\mathrm{Hz}$

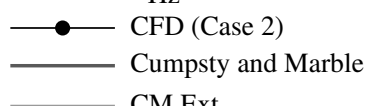
CM Ext. c) $w_{1}^{-} / w_{1}^{s}$

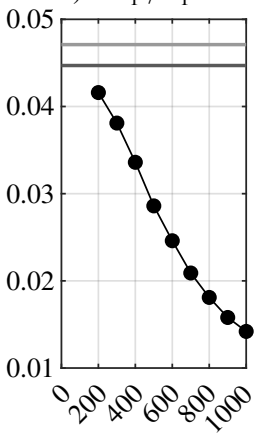

$\mathrm{Hz}$

Fig. 15 Validation of the compact model extension (CM Ext.) with the CFD results of Case 2 and the Cumpsty and Marble [5] model.

To further evaluate the performance of the CM Ext., Fig. 16 compares the entropy wave TF (Fig. 16a) and the entropy noise TFs (Figs. 16p and c) with the non-compact $200 \mathrm{~Hz}$ CFD results and the Cumpsty and Marble [5] model. If Fig. 16 was plotted with respect to the frequency as in Fig. 15, it could be seen that in all cases the CFD results are approaching the $0 \mathrm{~Hz}$ results of the $\mathrm{CM}$ Ext. The case comparison allows to see if the CM Ext. is capable of predicting the trend, where Case 0 has no cooling flow and Case 1-3 have an increasing cooling mass flow rate respectively. This essentially shows the impact of additional mass injection on the TFs. The trend of the entropy wave attenuation in Fig. 16a is very well predicted by the CM Ext. whereas the Cumpsty and Marble [5] model assumes entropy wave conservation. This is despite the fact that the CM Ext. assumes perfect mixing at the outlet (see Equation $8[10$, while film cooling is designed to stay on the blade surface and displace the hot combustion gasses. Though the assumption of perfect mixing is clearly not valid from a film cooling point of view, results show that this assumption is sufficient to provide qualitatively and quantitatively correct results. Considering the transmitted entropy noise TF in Fig. 16 , one can observe that the CM Ext. captures the trend well but provides a slightly higher TF in comparison to the Cumpsty and Marble [5] model. Here, is has to be mentioned that the Cumpsty and Marble [5] model accounts for the cooling flow by adjusting the outlet Mach number only and considers a mass flow rate of unity across the blade row, so that the effect of the cooling flow is modelled through a change in the geometry. The reflected entropy noise TF in Fig. 16: 
shows that the CM Ext. still captures the trend, but over predicts the TF by $6.9 \%$ with respect to the Cumpsty and Marble [5] model for Case 3. The CM Ext. assumes that all flow quantities are uniform along the $y$-direction at the inlet and outlet position. This is clearly not true in the case of film cooling. Hence, the locally generated entropy noise near the blade due to film cooling is not correctly accounted for. This may be the reason that the CM Ext. does not perfectly reproduce the acoustic TFs. However, the main advantage of the CM Ext. is the capability of capturing the attenuation of the entropy waves due to film cooling. This is very important for the prediction of entropy noise in consecutive blade rows. Mahmoudi et al. [9] showed that the entropy wave attenuation through a turbine stage needs to be considered in order to accurately predict the entropy noise generation. It can be seen in Fig. 16, that for all TFs for Case 0, the results of the CM Ext. collapse onto the original Cumpsty and Marble [5] model.

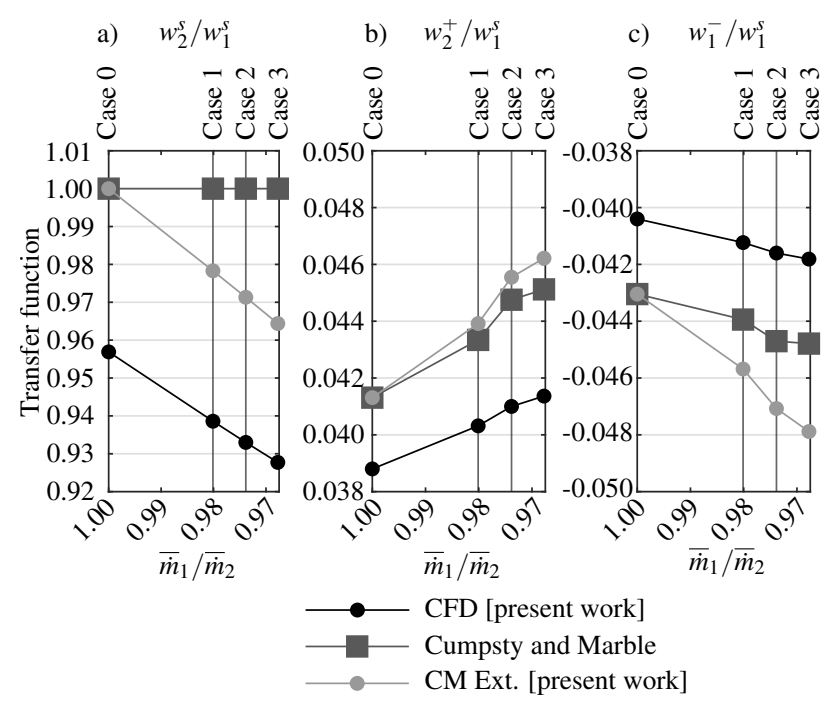

Fig. 16 Comparison of the CM Ext. with the CFD results (Case 0-3) and the Cumpsty and Marble [5] model with respect to the mass flow ratio $\overline{\dot{m}}_{1} / \overline{\dot{m}}_{2}$.

The influence of mass injection through a cooling flow is further investigated analytically. For this Eq. 18 is substituted into Eq. 20 and rearranged, leading to:

$$
\underbrace{\frac{\overline{\dot{m}}_{1}}{\overline{\dot{m}}_{2}}\left(\frac{\bar{s}_{1}}{\bar{s}_{2}}-1\right)}_{<<1 \text { because } \bar{s}_{1} \simeq \bar{s}_{2}} \frac{\dot{m}_{1}^{\prime}}{\overline{\dot{m}}_{1}}+\underbrace{\frac{\overline{\dot{m}}_{1}}{\overline{\dot{m}}_{2}} \frac{c_{p}}{\bar{s}_{2}}}_{\simeq 1} \frac{s_{1}^{\prime}}{c_{p}}=\frac{c_{p}}{\bar{s}_{2}} \frac{s_{2}^{\prime}}{c_{p}} .
$$

Since $\bar{s}_{1} \simeq \bar{s}_{2}$, the first term on the left hand side becomes much smaller than one, and the contribution of $\dot{m}_{1}^{\prime} / \overline{\dot{m}}_{1}$ (which includes acoustic fluctuations) on the downstream entropy perturbation $\left(s_{2}^{\prime} / c_{p}\right)$ is very small. This shows that the downstream entropy perturbation $\left(s_{2}^{\prime} / c_{p}\right)$ is driven by the upstream entropy perturbation $\left(s_{1}^{\prime} / c_{p}\right)$ and depends linearly on the mass flow ratio $\overline{\dot{m}_{1}} / \overline{\dot{m}}_{2}$. This result is in line with the observation of Fig. 16 , where the entropy wave attenuation is almost directly dependent on the mass flow ratio. Hence, the CM Ext. correctly captures the entropy wave attenuation despite the acoustic results being not perfect. The impact of mass injection on the acoustics is more complex. However, 
the fact that the original Cumpsty and Marble [5] model captures the trend very well in Fig. $16 \mathrm{~b}$ and $\mathrm{c}$ shows that the main factor is the global acceleration.

A parametric study to analyse the effect of the temperature ratio $\left(T R=T_{c} / T_{\infty}\right)$ has also been performed. In general, $T R$ values vary from 0.5 to 0.85 according to Han et al. [38], while the investigated range was extended (0.3 to 1.0$)$. For this purpose the inlet mass flow rate $\dot{m}_{1}$, inlet temperature $T_{1}$, and the coolant mass flow rate $\dot{m}_{c}$ were kept constant, while the coolant temperature $T_{c}$ has been varied to achieve a range of different $T R$. These boundary conditions resulted in a fixed mass flow ratio of $\bar{m}_{1} / \overline{\dot{m}}_{2}=0.953$, a nearly constant inlet Mach number and a slightly decreasing outlet Mach number with decreasing $T R$. The results at varying $T R$ are shown in Fig. 17

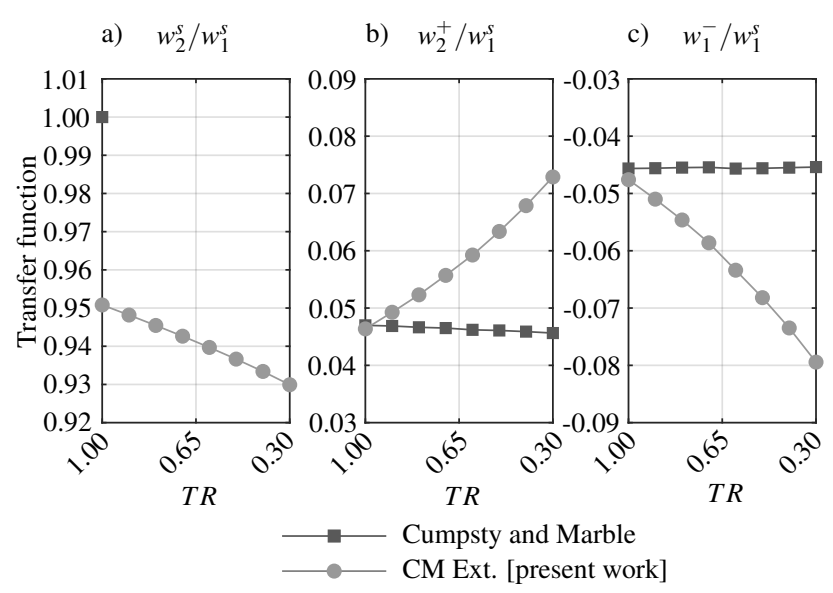

Fig. 17 Effect of temperature ratio on a) the entropy wave TF, b) the transmitted entropy noise TF and c) reflected entropy noise TF for the Cumpsty and Marble [5] model and the CM Ext.

$T R=1$ indicates isothermal cooling, where the coolant temperature is equal to the inlet temperature $\left(T_{c}=T_{1}\right)$. While this configuration does not provide any cooling benefits, it is interesting from an analytical standpoint. The comparison between the Cumpsty and Marble [5] model and the CM Ext. at $T R=1$ show that they almost collapse onto each other for the transmitted entropy noise TF in Fig. 17b, with a difference of 1.3\%. For the entropy wave TF and the reflected entropy noise TF (see Fig. $17 \mathrm{l}$ and c) the difference is $4.9 \%$ and $4.3 \%$ respectively. This difference can be closely correlated to the mass flow rate ratio of $\overline{\dot{m}}_{1} / \overline{\dot{m}}_{2}=0.953$. In addition, this good agreement between the entropy noise transfer functions evaluated by the Cumpsty and Marble [5] and CM Ext. models at isothermal conditions indicate that the assumption of no acoustic or entropy fluctuations introduced by the coolant flow is indeed reasonable.

As the coolant temperature is decreased, the $T R=T_{c} / T_{\infty}$ starts to decrease. This causes the entropy wave TF in Fig. 17 to further attenuate in a linear fashion, even though the inlet and coolant mass flow rates are constant. This is likely due to the change of the entropy ratio $\bar{s}_{1} / \bar{s}_{2}$, since all mass flow ratios in Eq. 34) stay constant. The impact of the $T R$ on the entropy noise TFs in Fig. 17p and $\mathrm{c}$ is more significant. As stated before, with the Cumpsty and Marble [5] model, the influence of the cooling on the acoustic transfer functions is only addressed through the changes in upstream and downstream Mach numbers. Mean flow variations being low in the present case, the Cumpsty and Marble model 
does not predict any significant variation in entropy noise generation as TR varies. On the other hand, CM Ext. predicts a noise increase for both transmitted and reflected waves when TR decreases. This point calls for further investigation.

\section{Conclusion}

As the indirect combustion noise is an increasingly important component of the total engine noise, it is vital to understand how different design aspects of a stator affect the indirect noise generation. It is necessary to account for acoustic effects when designing and optimising high pressure turbine film cooling systems for the next generation of high performance civil aeroengines. Along these lines, this study investigated the entropy noise generation using a URANS simulation of a small 3D blade mid-span section of a stator blade with film cooling on the leading edge, pressure side, and suction side of the blade.

While a frequency range of $200 \mathrm{~Hz}$ to $1000 \mathrm{~Hz}$ was investigated, the results showed that the entropy wave amplitude is only minimally attenuated by the injection of a cooling flow. However, the added mass flow rate changes the velocity field, and hence the bulk flow undergoes additional acceleration. The main acceleration of the mainstream and the local acceleration on the suction side of the turbine blade leads to an increase in the reflected and transmitted acoustic transfer function of up to $12 \%$, for an increase in mass flow rate of $3.4 \%$.

Furthermore, the low-order model of Cumpsty and Marble [5] for indirect entropy noise has been extended to incorporate the effect of film cooling flows. The evaluation of the CM Ext. showed that it captures the entropy wave attenuation due to film cooling across a single blade row very well. While the increase in entropy noise TFs is captured, the amplitude of the reflected entropy noise TF is over estimated. Hence, for practical applications a combination of the CM Ext. for the entropy wave attenuation and the Cumpsty and Marble [5] model for the acoustic TFs may be beneficial. The results of the CM Ext. also showed that increasing the temperature difference between the main and coolant flows further attenuated the entropy wave, which has beneficial consequences, as it will reduce the generation of entropy noise in downstream turbine stages. However, this increase in entropy wave attenuation is, to some extent, balanced by a local increase in entropy noise generation, that may in fact strengthen the impact of the noise as felt on the ground.

\section{Appendix}

This appendix covers the flow and temperature fields of film cooling obtained using the present URANS simulations. For the film cooling case (Case 1-3) there are no experimental measurements available on the PoliMi stator blade, as this turbine does not have any cooling flow. The film cooling performance was investigated numerically in this study by comparing three different cases. Case 2 refers to the BCs correlated with the $\mathrm{C} 3 \mathrm{X}$ test vane [23], while for Case 1 and Case 3 the cooling mass flow rate has been decreased and increased by 25\%, respectively. Hence, the cooling mass flow rate increases from Case 1 to Case 3. This changes the blowing ratio (Equation (3)) and the momentum flux ratio (Equation $(\mathrm{A}-1)$ ), impacting the cooling flow behaviour. The blowing ratio and the momentum flux ratio for each case 
Table A-1 Blowing ratios on the SH, PS and SS for Case 1-3.

\begin{tabular}{lccccccc}
\hline & & \multicolumn{3}{c}{$B R$} & & \multicolumn{3}{c}{$I$} \\
\cline { 3 - 8 } & $\dot{m}_{c}$ & SH & PS & SS & SH & PS & SS \\
\hline Case 3 & $+25 \%$ & 2.36 & 1.98 & 0.92 & 4.74 & 3.29 & 0.77 \\
Case 2 & $\approx \mathrm{C} 3 \mathrm{X}$ & 1.89 & 1.62 & 0.74 & 3.06 & 2.24 & 0.53 \\
Case 1 & $-25 \%$ & 0.64 & 0.72 & 0.55 & 0.41 & 0.45 & 0.30 \\
\hline
\end{tabular}

and location, namely SH, PS, and SS, are listed in Table A-1 To understand the characteristics of the different cooling flows the adiabatic effectiveness (Equation $(\mathrm{A}-2)$ ) is utilised.

$$
\begin{aligned}
I & =U_{c}^{2} \rho_{c} / U_{\infty}^{2} \rho_{\infty} \\
\eta & =\left(T_{\infty}-T_{a w}\right) /\left(T_{\infty}-T_{c, \text { exit }}\right)
\end{aligned}
$$

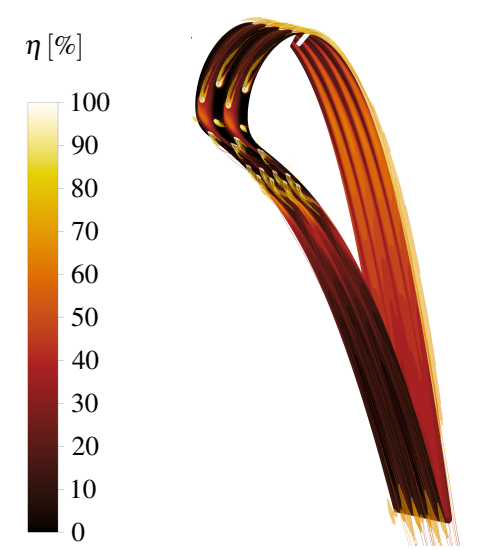

Fig. A-1 3D contour of the adiabatic effectiveness on the blade surface and coolant streamlines, (two spanwise instances).

It should be noted here that the term "detachment" refers to the cooling flow — whether it is attached to the blade surface. This is distinct from flow separation, as there was no flow separation observed in any of the cases tested here. A cooling flow can be detached if it penetrates the mainstream too far, thereby reducing its effectiveness. Thole et al. [39] showed that the momentum flux ratio is the scaling parameter that dictates whether the coolant flow stays attached or detaches. Results on a flat plate with a coolant injection angle of $35^{\circ}$ showed that the coolant flow stays attached if $I<0.4$, detaches and then reattaches if $0.4<I<0.8$ and fully detaches if $I>0.8$ [39]. The adiabatic effectiveness contour on the SS was compared for all cases in Fig. A-2.

In Case 2 (middle) the cooling flow detaches and then reattaches at approximately $x=16 \mathrm{~mm}$ (white X in Fig. A-2 at a momentum flux ratio of 0.53 . As the momentum flux ratio is increased to 0.77 in Case 3 , the coolant flow fully 
detaches and does not reattach, decreasing the adiabatic effectiveness with respect to Case 2. However, when the momentum flux ratio is decreased to 0.3 in Case 1, the coolant flow stays attached to the blade surface, spreading quickly along the blade span. However, the adiabatic effectiveness in Case 1 starts to decrease at approximately $x=17 \mathrm{~mm}$, yet it still outperforms the flow in Case 3. The flow detachment found on the SS in the simulation follows the momentum flux scaling proposed by Thole et al. [39]. The curvature of the blade creates a favourable pressure gradient which presses cooling flow to the blade surface. This was observed in Case 3 where the flow is fully detached, however stays near the blade, leading to a uniform adiabatic effectiveness of approximately $20 \%$.

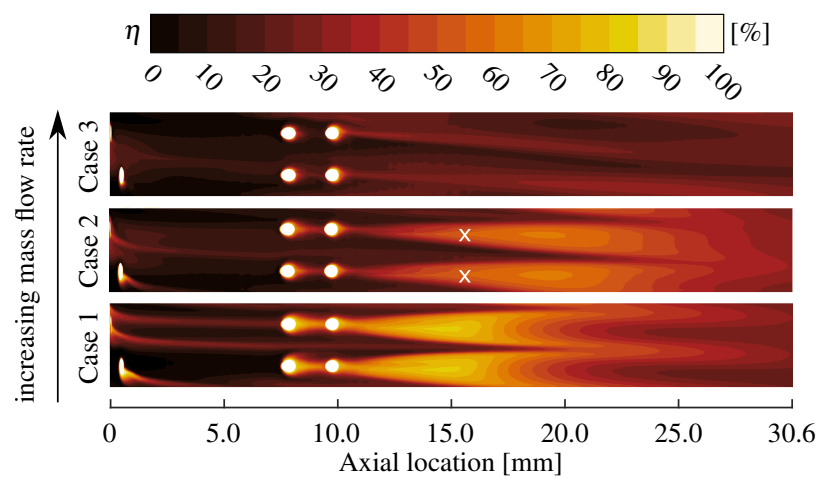

Fig. A-2 Contour of the adiabatic effectiveness on the SS of the stator blade for Case 1-3.

Looking at the adiabatic effectiveness around the stagnation point in the SH region in Fig. A-3, the cooling flow detaches in all cases, even for Case 1 with a momentum flux ratio of 0.41 . This separation is due to the low velocity of the mainstream flow in the stagnation region [40]. As the cooling flow penetrates the mainstream, it is pressed back onto the leading edge of the blade, creating a region of high adiabatic effectiveness (white rectangle). This region slightly increases in spanwise direction with increasing cooling mass flow but the effectiveness was reduced. Towards the SS, the adiabatic effectiveness increases with decreasing momentum flux ratio as the flow stays attached, however it is more localised and does not spread as much in spanwise direction. On the other hand, the adiabatic effectiveness decreases from the SH towards the PS for decreasing momentum flux ratios. For Case 1, the flow stays attached, $I_{S H}=0.4$, however it does not spread much in the spanwise direction between the SH and the PS cooling holes. While for $I_{S H}=3$ (Case 2) and $I_{S H}=4.7$ (Case 3) the flow fully detaches, it interacts further downstream with the coolant from the first row on the PS, creating a distinctive spanwise increase in adiabatic effectiveness shown in Fig. A-3 (white ellipse). This is consistent with the findings by Ito et al. [41], who showed that for concave surfaces the adiabatic effectiveness increases with increasing blowing ratio some distance downstream of the hole.

On the PS of the blade, Fig. A-3 shows that the adiabatic effectiveness drops significantly in comparison to the SS. This is partially due to the fact that approximately $60 \%$ less coolant was injected in comparison to the SS in order to meet the PS blowing ratio for Case 2. The low freestream velocity of $\sim 40 \mathrm{~m} / \mathrm{s}$ at the PS hole outlet means that the velocity of the coolant $\left(U_{c}\right)$ has to be proportionally low to meet the BR, resulting in a low cooling mass flow on the 
PS. At the same time this led to high momentum flux ratio values of 2.2 and 3.3 for Case 2 and 3, respectively, and caused the coolant to separate. In Case 1, a better cooling coverage is achieved, especially towards the trailing edge as $I_{P S}=0.45$. The interaction of the SH and PS clearly shows an improvement in adiabatic effectiveness. The increase of adiabatic effectiveness due to the interaction between neighbouring coolant jets has been shown previously [29].

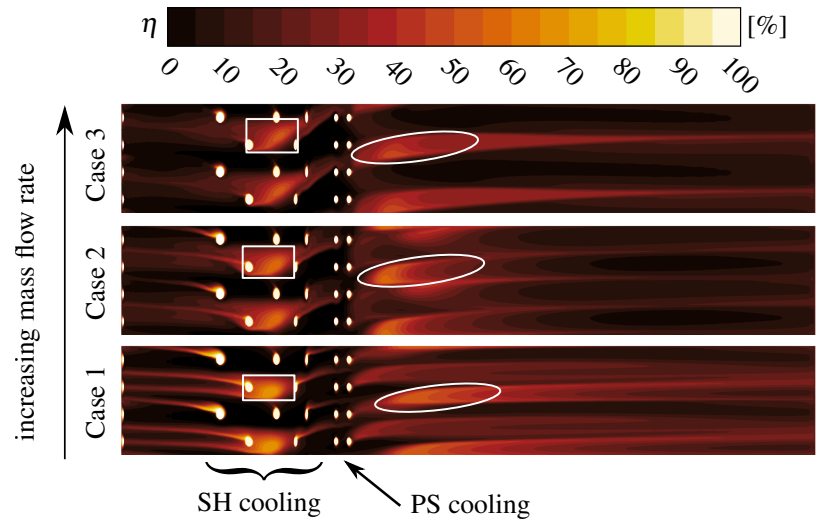

Fig. A-3 Contour of the adiabatic effectiveness of the SH and the PS of the stator blade for Case 1-3.

The analysis of the film cooling geometry of the C3X vane applied to the PoliMi stator blade showed that on this specific blade, the film cooling geometry is not optimised. The pairs of cooling rows on the SS and PS are likely to be more efficient if they were staggered rather than being in line. Since the individual coolant jets would interact and shield effectively the blade surface from the mainstream flow. The same is true for the SH array of holes. Since the leading edge radius is small, a pitch to diameter ratio of 4.0 is rather large, as the ratio is commonly between 3.0 and 4.0 (see Table 1). The spacing to diameter ratio of 7.5 is also towards the upper limit of 3.0-8.0 and prevents the interaction between the cooling flows. This SH arrangement leaves large areas unprotected from the mainstream temperature. Nevertheless, the focus of this study is on the entropy and acoustic analysis rather than maximising the cooling effectiveness, so this cooling arrangement is considered satisfactory. The optimisation of film cooling layouts for both aerothermal and acoustic efficiency are not explored in this paper.

\section{Acknowledgments}

The authors would like to thank Prof. P. Gaetani from the Politecnico di Milano for sharing the geometry and experimental data from their high-pressure turbine. This work was supported by the Engineering and Physical Sciences Research Council (EPSRC) [grant number EP/N509541/1].

\section{References}

[1] Scherer, C., "Global Market Forecast Cities, Airports \& Aircraft 2019-2038," Tech. rep., Airbus, 2019. URL https: //wWw.airbus.com/aircraft/market/global-market-forecast.html 
[2] Dowling, A. P., and Mahmoudi, Y., "Combustion noise," Proceedings of the Combustion Institute, Vol. 35, No. 1, 2015, pp. 65-100. https://doi.org/10.1016/j.proci.2014.08.016

[3] Bragg, S., "Combustion Noise," Journal of the Institute Fuel, Vol. 36, 1963, pp. 12-16.

[4] Marble, F., and Candel, S., “Acoustic disturbance from gas non-uniformities convected through a nozzle,” Journal of Sound and Vibration, Vol. 55, No. 2, 1977, pp. 225-243. https://doi.org/10.1016/0022-460X(77)90596-X

[5] Cumpsty, N. A., and Marble, F. E., "The interaction of entropy fluctuations with turbine blade rows; a mechanism of turbojet engine noise," Proceedings of the Royal Society of London. A. Mathematical and Physical Sciences, Vol. 357, No. 1690, 1977, pp. 323-344. https://doi.org/10.1098/rspa.1977.0171.

[6] Ihme, M., “Combustion and Engine-Core Noise,” Annual Review of Fluid Mechanics, Vol. 49, No. 1, 2017, pp. 277-310. https://doi.org/10.1146/annurev-fluid-122414-034542

[7] Magri, L., O’Brien, J., and Ihme, M., "Effects of Nozzle Helmholtz Number on Indirect Combustion Noise by Compositional Perturbations," Journal of Engineering for Gas Turbines and Power, Vol. 140, No. 3, 2018, pp. 1-9. https://doi.org/10.1115/1. 4037914

[8] Mahmoudi, Y., Dowling, A. P., and Stow, S. R., "Acoustic and Entropy Waves in Nozzles in Combustion Noise Framework," AIAA Journal, Vol. 55, No. 7, 2017, pp. 2369-2381. https://doi.org/10.2514/1.J055597

[9] Mahmoudi, Y., Giusti, A., Mastorakos, E., and Dowling, A. P., "Low-Order Modeling of Combustion Noise in an Aero-Engine: The Effect of Entropy Dispersion,” Journal of Engineering for Gas Turbines and Power, Vol. 140, No. 1, 2018, pp. 1-7. https://doi.org/10.1115/1.4037321

[10] Mishra, A., and Bodony, D. J., "Evaluation of actuator disk theory for predicting indirect combustion noise," Journal of Sound and Vibration, Vol. 332, No. 4, 2013, pp. 821-838. https://doi.org/10.1016/j.jsv.2012.09.025.

[11] Leyko, M., Duran, I., Moreau, S., Nicoud, F., and Poinsot, T., "Simulation and modelling of the waves transmission and generation in a stator blade row in a combustion-noise framework," Journal of Sound and Vibration, Vol. 333, No. 23, 2014, pp. 6090-6106. https://doi.org/10.1016/j.jsv.2014.06.034

[12] Bauerheim, M., Duran, I., Livebardon, T., Wang, G., Moreau, S., and Poinsot, T., "Transmission and reflection of acoustic and entropy waves through a stator-rotor stage," Journal of Sound and Vibration, Vol. 374, 2016, pp. 260-278. https: //doi.org/10.1016/j.jsv.2016.03.041

[13] Morgans, A. S., Goh, C. S., and Dahan, J. A., "The dissipation and shear dispersion of entropy waves in combustor thermoacoustics," Journal of Fluid Mechanics, Vol. 733, No. R2, 2013, p. R2. https://doi.org/10.1017/jfm.2013.448

[14] Giusti, A., Worth, N. A., Mastorakos, E., and Dowling, A. P., "Experimental and Numerical Investigation into the Propagation of Entropy Waves,” AIAA Journal, Vol. 55, No. 2, 2017, pp. 446-458. https://doi.org/10.2514/1.J055199 
[15] Fattahi, A., Hosseinalipour, S. M., and Karimi, N., "On the dissipation and dispersion of entropy waves in heat transferring channel flows," Physics of Fluids, Vol. 29, No. 8, 2017, p. 087104. https://doi.org/10.1063/1.4999046

[16] Papadogiannis, D., Wang, G., Moreau, S., Duchaine, F., Gicquel, L., and Nicoud, F., “Assessment of the Indirect Combustion Noise Generated in a Transonic High-Pressure Turbine Stage," Journal of Engineering for Gas Turbines and Power, Vol. 138, No. 4, 2016, p. 041503. https://doi.org/10.1115/1.4031404

[17] Ceci, A., Gojon, R., and Mihaescu, M., "Large Eddy Simulations for Indirect Combustion Noise Assessment in a Nozzle Guide Vane Passage," Flow, Turbulence and Combustion, Vol. 102, No. 2, 2019, pp. 299-311. https://doi.org/10.1007/s10494-0189964-9

[18] Becerril, C., Moreau, S., and Gicquel, L. Y. M., "Study of Combustion Noise Generation in a Realistic Turbine Stage Configuration," Volume 2B: Turbomachinery, American Society of Mechanical Engineers, 2018. https://doi.org/10.1115/ GT2018-75062.

[19] Knobloch, K., Guerin, S., Holewa, A., Mahmoudi-Larimi, Y., Hynes, T., and Bake, F., "Noise Transmission Characteristics of a High Pressure Turbine Stage," 22nd AIAA/CEAS Aeroacoustics Conference, American Institute of Aeronautics and Astronautics, Reston, Virginia, 2016, pp. 1-12. https://doi.org/10.2514/6.2016-3001

[20] Xu, L., Bo, S., Hongde, Y., and Lei, W., "Evolution of Rolls-royce Air-cooled Turbine Blades and Feature Analysis," Procedia Engineering, Vol. 99, 2015, pp. 1482-1491. https://doi.org/10.1016/j.proeng.2014.12.689.

[21] Knobloch, K., Werner, T., and Bake, F., "Entropy Noise Generation and Reduction in a Heated Nozzle Flow," 21st AIAA/CEAS Aeroacoustics Conference, American Institute of Aeronautics and Astronautics, Reston, Virginia, 2015. https://doi.org/10.2514/6.2015-2818

[22] Gaetani, P., and Persico, G., "Transport of Entropy Waves Within a High Pressure Turbine Stage,” Journal of Turbomachinery, Vol. 141, No. 3, 2019, p. 031006. https://doi.org/10.1115/1.4042165.

[23] Hylton, L. D., Nirmalan, V., Sultanian, B. K., and Kaufman, R. M., "The Effects of Leading Edge and Downstream on Turbine Vane Heat Transfer,” NASA Contractor Report 182133, 1988.

[24] Knobloch, K., Neuhaus, L., Bake, F., Gaetani, P., and Persico, G., "Experimental Assessment of Noise Generation and Transmission in a High-Pressure Transonic Turbine Stage,” Journal of Turbomachinery, Vol. 139, No. 10, 2017, pp. 1-12. https://doi.org/10.1115/1.4036344

[25] Persico, G., Mora, A., Gaetani, P., and Savini, M., "Unsteady Aerodynamics of a Low Aspect Ratio Turbine Stage: Modeling Issues and Flow Physics,” Journal of Turbomachinery, Vol. 134, No. 6, 2012, p. 061030. https://doi.org/10.1115/1.4004021.

[26] Gaetani, P., Persico, G., and Osnaghi, C., "Effects of Axial Gap on the Vane-Rotor Interaction in a Low Aspect Ratio Turbine Stage," Journal of Propulsion and Power, Vol. 26, No. 2, 2010, pp. 325-334. https://doi.org/10.2514/1.37616. 
[27] Bassi, F., Fontaneto, F., Franchina, N., Ghidoni, A., and Savini, M., "Turbine vane film cooling: Heat transfer evaluation using high-order discontinuous Galerkin RANS computations," International Journal of Heat and Fluid Flow, Vol. 61, 2016, pp. 610-625. https://doi.org/10.1016/j.ijheatfluidflow.2016.07.007

[28] Borello, D., D’Angeli, L., Salvagni, A., Venturini, P., and Rispoli, F., "Study of Particles Deposition in Gas Turbine Blades in Presence of Film Cooling," Volume 5B: Heat Transfer, American Society of Mechanical Engineers, 2014. https://doi.org/10.1115/GT2014-26250

[29] Bogard, D. G., and Thole, K. A., “Gas Turbine Film Cooling,” Journal of Propulsion and Power, Vol. 22, No. 2, 2006, pp. 249-270. https://doi.org/10.2514/1.18034

[30] Bach, T., Mahmoudi, Y., and Spence, S., "Unsteady RANS Simulation on the Effect of Film Cooling on Entropy Noise Generation in a Two- Dimensional Stator Cascade," 26th International Congress on Sound and Vibration, International Institute of Acoustics and Vibration (IIAV), Montréal, 2019, pp. 1-8.

[31] Bake, F., Richter, C., Mühlbauer, B., Kings, N., Röhle, I., Thiele, F., and Noll, B., “The Entropy Wave Generator (EWG): A reference case on entropy noise," Journal of Sound and Vibration, Vol. 326, No. 3-5, 2009, pp. 574-598. https://doi.org/10.1016/j.jsv.2009.05.018

[32] Menter, F. R., “Two-equation eddy-viscosity turbulence models for engineering applications," AIAA Journal, Vol. 32, No. 8, 1994, pp. 1598-1605. https://doi.org/10.2514/3.12149

[33] Barth, T., and Jespersen, D., "The design and application of upwind schemes on unstructured meshes," 27th Aerospace Sciences Meeting, American Institute of Aeronautics and Astronautics, Reston, Virigina, 1989. https://doi.org/10.2514/6.1989-366.

[34] ANSYS, C., 18.2: CFX Modelling Guide \& Theory Guide, ANSYS Help Viewer, 2017.

[35] Stewart, W. R., and Bogard, D. G., "Experimental Thermal Field Measurements of Film Cooling Above the Suction Surface of a Turbine Vane," Journal of Engineering for Gas Turbines and Power, Vol. 137, No. 10, 2015. https://doi.org/10.1115/1.4030263

[36] Emmanuelli, A. B., Huet, M., Le Garrec, T., and Ducruix, S., "Study of Entropy Noise through a 2D Stator using CAA," 2018 AIAA/CEAS Aeroacoustics Conference, American Institute of Aeronautics and Astronautics, Reston, Virginia, 2018. https://doi.org/10.2514/6.2018-3915

[37] Ullrich, W. C., Mahmoudi, Y., Lackhove, K., Fischer, A., Hirsch, C., Sattelmayer, T., Dowling, A. P., Swaminathan, N., Sadiki, A., and Staufer, M., "Prediction of Combustion Noise in a Model Combustor Using a Network Model and a LNSE Approach," Journal of Engineering for Gas Turbines and Power, Vol. 140, No. 4, 2018, pp. 1-13. https://doi.org/10.1115/1.4038026

[38] Han, J.-C., Dutta, S., and Ekkad, S., Gas Turbine Heat Transfer and Cooling Technology, $2^{\text {nd }}$ ed., CRC Press, 2012. https://doi.org/10.1201/b13616 
[39] Thole, K., Sinha, A., Bogard, D., and Crawford, M., "Mean temperature measurements of jets with a crossflow for gas turbine film cooling application," Third International Symposium on Transport Phenomena and Dynamics of Rotating Machinery (ISROMAC-3), , No. January 1992, 1990, pp. 69-86.

[40] Cutbirth, J. M., and Bogard, D. G., "Thermal Field and Flow Visualization Within the Stagnation Region of a Film-Cooled Turbine Vane," Journal of Turbomachinery, Vol. 124, No. 2, 2002, pp. 200-206. https://doi.org/10.1115/1.1451086

[41] Ito, S., Goldstein, R. J., and Eckert, E. R. G., "Film Cooling of a Gas Turbine Blade," Journal of Engineering for Power, Vol. 100, No. 3, 1978, pp. 476-481. https://doi.org/10.1115/1.3446382 